\title{
Deficiency and Insufficiency of Vitamin D in Women of Childbearing Age: A Systematic Review and Meta-analysis
}

\section{Deficiência e insuficiência de vitamina $D$ em mulheres na idade reprodutiva: Uma revisão sistemática e meta-análise}

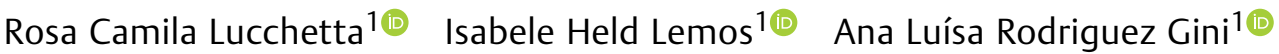 \\ Sophia de Andrade Cavicchioli ${ }^{20}$ Marcela Forgerini ${ }^{10}$ Fabiana Rossi Varallo ${ }^{30}$ \\ Mariane Nunes de Nadai ${ }^{4}$ Fernando Fernandez-Llimos ${ }^{5}{ }^{\circledR}$ Patricia de Carvalho Mastroianni ${ }^{1}{ }^{\circledR}$
}

\footnotetext{
1 Department of Drugs and Medicines, School of Pharmaceutical

Sciences, Universidade Estadual de São Paulo, São Paulo, SP, Brazil

2 Universidade de Araraquara, Araraquara, São Paulo, SP, Brazil

3 Department of Pharmaceutical Sciences, Faculty of Pharmaceutical Sciences of Ribeirão Preto, Universidade de São Paulo, Ribeirão Preto, SP, Brazil

${ }^{4}$ Department of Dentistry, Pediatric Dentistry and Public Health, Bauru School of Dentistry, Universidade de São Paulo, Bauru, SP, Brazil

${ }^{5}$ Department of Drug Sciences, Laboratory of Pharmacology, Faculty of Pharmacy, Universidade do Porto, Porto, Portugal
}

\author{
Address for correspondence Patricia de Carvalho Mastroianni, PhD, \\ Department of Drugs and Medicines, School of Pharmaceutical \\ Sciences, São Paulo State University (UNESP) Highway Jaú, Km 01 s/n, \\ 14800-901, Campus Vile, Araraquara, SP, Brazil \\ (e-mail: patricia.mastroianni@unesp.br).
}

Rev Bras Ginecol Obstet 2022;44(4):409-424.

\section{Abstract \\ Keywords \\ - cholecalciferol \\ - vitamin D deficiency \\ - nutritional epidemiology \\ - maternal nutrition \\ - women's health}

Objective To estimate the prevalence of inadequate vitamin D level and its associated factors for women of childbearing age in Brazil.

Methods A systematic review was conducted (last updated May 2020). Meta-analyses were performed using the inverse-variance for fixed models with summary proportion calculation by Freeman-Tukey double arcsine. Reporting and methodological quality were assessed using the Joanna Briggs Institute tool for prevalence studies.

Results Our review identified 31 studies, comprising 4,006 participants. All the studies had at least one weakness, mainly due to the use of convenience sampling and small sample size. The overall prevalence of vitamin D deficiency, insufficiency, and both deficiency and insufficiency were 35\% (confidence interval, 95\%Cl: 34-37\%), 42\% (95\%Cl: $41-44 \%)$, and $72 \%$ (95\% Cl: $71-74 \%)$, respectively.

Conclusion Although the magnitude of the prevalence of inadequate levels of vitamin $D$ is uncertain, the evidence suggests that presence of vitamin $D$ deficiency or insufficiency in women of reproductive age can cause moderate to severe problems. received

April 20, 2021

accepted

November 3, 2021

published online

February 24, 2022
DOI https://doi.org/ 10.1055/s-0042-1742409. ISSN 0100-7203. (c) 2022. Federação Brasileira de Ginecologia e Obstetrícia. All rights reserved.

This is an open access article published by Thieme under the terms of the Creative Commons Attribution License, permitting unrestricted use, distribution, and reproduction so long as the original work is properly cited. (https://creativecommons.org/licenses/by/4.0/)

Thieme Revinter Publicações Ltda., Rua do Matoso 170, Rio de Janeiro, RJ, CEP 20270-135, Brazil 


\section{Resumo}

$$
\begin{aligned}
& \text { Palavras-chave } \\
& \text { - colecalciferol } \\
& \text { - deficiência de } \\
& \text { vitamina D } \\
& \text { - epidemiologia } \\
& \text { nutricional } \\
& \text { - nutrição materna } \\
& \text { - saúde da mulher }
\end{aligned}
$$

Objetivo Estimar a prevalência de níveis inadequados de vitamina $D$ e seus fatores associados para mulheres em idade fértil no Brasil.

Métodos Uma revisão sistemática foi realizada (última atualização em maio de 2020). As meta-análises foram realizadas usando o inverso da variância para o modelo fixo com cálculo de proporção sumarizada por transformação arco-seno duplo de Freeman-Tukey. A qualidade metodológica e de reporte foi avaliada usando a ferramenta do Joanna Briggs Institute para estudos de prevalência.

Resultados Nossa revisão identificou 31 estudos, compreendendo 4.006 participantes. Todos os estudos apresentaram pelo menos uma limitação, principalmente devido ao uso de amostra de conveniência e tamanho amostral pequeno. As prevalências gerais de deficiência, insuficiência e deficiência de vitamina D foram 35\% (intervalo de confiança, IC 95\%: 34-37\%), 42\% (IC 95\%: 41-44\%) e 72\% (IC 95\%: 71-74\%), respectivamente.

Conclusão Embora a magnitude da prevalência de níveis inadequados de vitamina $D$ seja incerta, a evidência sugere que presença de deficiência ou insuficiência de vitamina $\mathrm{D}$ em mulheres em idade reprodutiva pode causar problemas moderados a graves.

\section{Introduction}

The deficiency and insufficiency of 25-hydroxyvitamin D, also known as $25(\mathrm{OH}) \mathrm{D}$ or vitamin $\mathrm{D}$, is a worldwide issue: less than $50 \%$ of the world population has an adequate level of vitamin $\mathrm{D}$, but in older people, pregnant women, and nonWestern immigrants the proportion is smaller. ${ }^{1}$ In pregnant women, for instance, the prevalence of insufficiency $(25(\mathrm{OH})$ $\mathrm{D}<50 \mathrm{nmol} / \mathrm{L})$ and deficiency $(25(\mathrm{OH}) \mathrm{D}<25 \mathrm{nmol} / \mathrm{L})$ ranged from $46 \%$ to $87 \%$ and $9 \%$ to $79 \%$, respectively. ${ }^{2}$ Even in warmer countries, such as Brazil, there is an alarming prevalence of vitamin D deficiency (28\%) and insufficiency (45\%), reaching $85 \%$ in pregnant women. ${ }^{3,4}$

Recent studies suggested that vitamin D homeostasis may be important for several nonskeletal outcomes, including cardiovascular and respiratory diseases, neuromuscular function, psoriasis, falls, obesity, type 2 diabetes mellitus, multiple sclerosis, colorectal cancer, and coronavirus disease 19 (COVID-19). ${ }^{5-12}$ Vitamin D deficiency also causes a series of poor gestational outcomes, ${ }^{13}$ increasing the risk of preeclampsia and gestational diabetes mellitus, as well as the production of maternal inflammatory cytokines, ${ }^{13,14}$ insulin resistance, ${ }^{13,15}$ and postpartum depression. ${ }^{13,16}$

In Brazil, there is a great variability in studies assessing insufficiency and deficiency of vitamin $D$ in women of childbearing age (12-68\%), ${ }^{17-19}$ but there is also a lack of evidence that systematically summarizes their prevalence. A systematic review (2019) evaluated the deficiency and insufficiency of vitamin D in Brazil, with no specific analysis for women of childbearing age. ${ }^{4}$ The present systematic review aimed to identify the prevalence and factors associated with inadequate levels of vitamin D in women of childbearing age in Brazil.

\section{Methods}

\section{Study Design, Protocol, and Registration}

A systematic review was performed in accordance with the Meta-analysis of Observational Studies in Epidemiology
(MOOSE) group, ${ }^{20}$ and Joanna Briggs Institute recommendations, ${ }^{21}$ and reported following the Preferred Reporting Items for Systematic Reviews and Meta-Analyses (PRISMA). ${ }^{22}$ The protocol of this review is available at Center for Open Science ${ }^{23}$ and PROSPERO (CRD42020221605). This study is part of a larger project that evaluated vitamins A, B, C, D, and E, calcium, iodine, iron, and zinc deficiencies in women of childbearing age in Brazil.

\section{Information Sources, Search Strategy, and Eligibility Criteria}

Electronic searches were conducted in the following databases: PubMed, Scopus, LILACS, World Health Organization (WHO), and CAPES' dissertations and theses (gray literature). The selection of these sources ensured including EMBASE, Medline, open access sources, scientific websites, and gray literature, ${ }^{24}$ through a predefined search strategy (available in the protocol) ${ }^{23}$ from their inception to May 2020. An additional manual search was performed using reference lists of reviews and included studies.

Studies that fulfilled the following criteria according to the CoCoPop acronym were included ${ }^{25}:$ i) Condition: vitamin D deficiency or insufficiency; ii) Context: Brazil, without restriction of setting; iii) Population: women of childbearing age (15-49 years old) without any restriction of diseases or physiological status (e.g., nonpregnant, pregnant, postpartum). Data from studies that reported the deficiencies of interest, using a different population classification (e.g., premenopausal women), or different laboratory parameters were separated for appropriate subgroup analyses. All types of articles were included, except for reviews, letters, comments, case reports, and case series. No language restriction was applied.

\section{Study Selection and Data Extraction}

Two researchers screened the titles and abstracts and evaluated the full-text articles independently. Discrepancies were 
solved in consensus meetings using another researcher as a referee.

Five researchers independently extracted the following data:

(i) Study characteristics (e.g., type of study, analysis period, state, region, funding, micronutrient assessed, and sampling method);

(ii) Participant characteristics (e.g., pregnant women, ethnicity, comorbidities, drug therapy or supplement in use, body mass index, age, education, per capita income);

(iii) Prevalence estimate, according to cutoff values used ( $\mathrm{n} / \mathrm{N}[\%]$ ) to total population and subgroups, when the information was available. When the studies reported vitamin D deficiency and insufficiency separately, we deduced the estimates considering the sum of participants.

\section{Synthesis of Results}

Although predefined cutoffs for the assessment of deficiencies and insufficiencies of vitamin D were not considered inclusion criteria in the present review, only studies that considered identical cutoffs were grouped.

The data synthesis was primarily done by meta-analysis. Transitivity assessment was performed by comparing the CoCoPop acronym for each study. ${ }^{25}$ Once important discrepancies were identified, sensitivity analyses with the exclusion of the study in question were performed (i.e., leave-oneout method). Proportion meta-analyses were conducted in the RStudio IDE (RStudio, PBC. Boston, MA, USA) software, version 3.6.3, $1.2 .5033,{ }^{26}$ using the READR (RStudio, PBC.) ${ }^{27}$ and META packages (RStudio, PBC.). ${ }^{28}$

In the base-case, direct proportion meta-analyses were conducted using the inverse variance method. ${ }^{28}$ To calculate the weighted summary proportion, the Freeman-Tukey double arcsine (PFT) was considered in the fixed effects model. ${ }^{22,28}$ Although high heterogeneity is expected and, therefore, a random effects model could be considered appropriate, a fixed effects model is preferred for the assessment of prevalence, because otherwise the weighting will not properly consider the weight of the studies. ${ }^{29}$ The result of the meta-analysis was given by the proportion combined with $95 \%$ confidence interval $(95 \% \mathrm{CI})$, as well as the list of proportions (presented as a percentages), with their respective $95 \%$ CIs found in the individual studies. A Higgins inconsistency test $\left(\mathrm{I}^{2}\right)$ with an estimator for $\operatorname{tau}^{2}$ was considered using the DerSimonian-Laird method.

Cumulative meta-analyses were also performed to assess changes and trends over time, and to highlight emerging or decreasing deficiency or insufficiency. Potential publication bias was assessed using rank tests with at least ten studies by meta-analysis. ${ }^{28}$

Sensitivity analyses were performed by the leave-one-out method. Subgroup and meta-regression analyses, considering the publication year, state and region of Brazil, comorbidities, age, or status (i.e., not pregnant, pregnant, postpartum) were planned for meta-analyses with at least ten studies. Alternative statistical methods were also con- ducted to validate the conclusions (i.e., GLMM, Logit transformation, random effects, and Hartung and Knapp for random models).

\section{Methodological Quality in Individual Studies}

An assessment of methodological and reporting quality based on the JBI Critical Appraisal Checklist for studies reporting prevalence data was conducted. ${ }^{30,31}$ Two reviewers performed the assessment, independently. In the absence of consensus, points of disagreement were resolved by a third investigator.

\section{Data Sharing and Data Accessibility}

The data that support the findings of this study are openly available in OSF at http:doi.org/10.17605/OSF.IO/J9QMH. ${ }^{23}$

\section{Results}

Our systematic review identified 1,977 records in the electronic databases after duplicate removal (PubMed, LILACS, and Scopus) and 91 additional records identified through other sources (manual search, WHO, and CAPES' dissertations and theses databases). After selection process, 31 studies were included, published between 2008 and 2020, reporting deficiency or insufficiency of vitamin $D$. The list of included and excluded studies, as well as a PRISMA flowchart, are available in the OSF. ${ }^{23}$ Of the 31 studies selected, 23 were cross-sectional, 4 prospective, 2 retrospective cohorts, and 2 were case-control studies. The studies were conducted between 1995 and 2017 (six studies did not report inclusion period), in cities in the Southeast $(n=18)$, South $(n=7)$, Northeast $(n=5)$, and Center-west $(n=2)$ Brazilian regions, with women selected mainly from outpatient care $(n=20)$. Araújo et al., ${ }^{32}$ Queiroz,, ${ }^{33}$ Queiroz et al., ${ }^{34}$ de Oliveira et al., ${ }^{35}$ and dos Santos et al. ${ }^{36}$ used a random probabilistic sampling, while Martins et al. $^{37}$ used convenience sampling (-Chart 1). . $^{3,17-19,32-68}$

Most studies assessed women of childbearing age $(n=13)$, followed by pregnant women $(n=10)$, adolescents $(n=6)$, and postpartum women $(n=4)$. Two studies assessed pregnant and nonpregnant women, concomitantly. Therefore, 4,006 participants were included, mainly women of childbearing age $(n=1,239)$, with a mean age ranged from 13 to 46 years old, and mean body mass index ranged from 22 to $46 \mathrm{~kg} / \mathrm{m}^{2}$. The majority of studies included women with a medical condition (e.g., HIV + , gestational diabetes mellitus, hypertension) or post Roux-en-Y gastric bypass surgery (RYGB, $n=18$ ). Although drug therapy use was not reported is most studies, nutrient supplementation $(n=11)$ or no supplementation $(n=11)$ use were reported. The main characteristics of the participants are described in - Chart 2. ${ }^{3,17-19,32-35,37-66}$

In the quality assessment, all studies had at least one 'No' answer, which suggests an overall poor reporting or methodological quality. The main questions with 'No' answers were regarding sample size $(n=30)$ and sampling method $(n=29)$. Questions with 'Yes' answers were about sample frame and valid methods used for the identification of the 
412 Deficiency and Insufficiency of Vitamin D in Women of Childbearing Age Lucchetta et al.

Chart 1 Description of the characteristics of the included studies

\begin{tabular}{|c|c|c|c|c|c|}
\hline Study & Inclusion period & $\begin{array}{l}\text { State/ } \\
\text { region }\end{array}$ & Setting & Cutoff values & Funding \\
\hline \multicolumn{6}{|l|}{ Cross-sectional studies } \\
\hline $\begin{array}{l}\text { Araújo et al. (2017), }{ }^{32} \\
\text { Queiroz (2016), }{ }^{33} \text { and } \\
\text { Queiroz et al. (2019) }\end{array}$ & Jun-Aug 2015 & $\mathrm{~PB} / \mathrm{NE}$ & School & $<75 \mathrm{nmol} / \mathrm{L}$ & NR \\
\hline Chrisostomo et al. $(2018)^{38}$ & \multicolumn{2}{|c|}{ Jan-Mar or Jul-Aug 2016 PR/S } & \multicolumn{2}{|c|}{$\begin{aligned} \text { Obstetrical care } & <75 \mathrm{nmol} / \mathrm{L} \\
& 50-75 \mathrm{nmol} / \mathrm{L} \\
& <50 \mathrm{nmol} / \mathrm{L}\end{aligned}$} & NR \\
\hline $\begin{array}{l}\text { Duran de Campos et al. } \\
(2008)^{39}\end{array}$ & Oct 1995-Jan 1999 & $\mathrm{SP} / \mathrm{SE}$ & Outpatient & $\begin{array}{l}25-50 \mathrm{nmol} / \mathrm{L} \\
12.5-25 \mathrm{nmol} / \mathrm{L}\end{array}$ & NR \\
\hline de Oliveira et al. $(2020)^{35}$ & Feb 2013-Nov 2014 & $\begin{array}{l}\text { DF, RJ, RS, } \\
\text { SC/S, SE, CW }\end{array}$ & School & $\begin{array}{l}<75 \mathrm{nmol} / \mathrm{L} \\
50-75 \mathrm{nmol} / \mathrm{L} \\
<50 \mathrm{nmol} / \mathrm{L}\end{array}$ & $\begin{array}{l}\text { Brazilian Funding } \\
\text { Authority for Studies and } \\
\text { Projects, and CNPq }\end{array}$ \\
\hline Souza et al. $(2019)^{40}$ & Jan-Feb 2017 & $\mathrm{MA} / \mathrm{NE}$ & Outpatient & $\begin{array}{l}<75 \mathrm{nmol} / \mathrm{L} \\
50-75 \mathrm{nmol} / \mathrm{L} \\
<50 \mathrm{nmol} / \mathrm{L}\end{array}$ & NR \\
\hline Delmonico et al. $(2018)^{41}$ & 2008-2016 & $\mathrm{RJ} / \mathrm{SE}$ & Outpatient & $<75 \mathrm{nmol} / \mathrm{L}$ & CAPES \\
\hline Prado et al. $(2015)^{3}$ & Dec 2011-Nov 2012 & $\mathrm{MG} / \mathrm{SE}$ & \multicolumn{2}{|c|}{ Obstetrical care $<50 \mathrm{nmol} / \mathrm{L}$} & FAPEMIG \\
\hline Ferreira et al. $(2015)^{42}$ & NR & $\mathrm{RJ} / \mathrm{SE}$ & Outpatient & $<50 \mathrm{nmol} / \mathrm{L}$ & FAPERJ \\
\hline Flauzino et al. $(2017)^{43}$ & Jul 2010-Mar 2011 & $\mathrm{PR} / \mathrm{S}$ & Outpatient & $<75 \mathrm{nmol} / \mathrm{L}$ & CAPES, CNPq, and UEL \\
\hline Lopes et al. $(2015)^{44}$ & 2011-2013 & $\mathrm{SP} / \mathrm{SE}$ & Outpatient & $<75 \mathrm{nmol} / \mathrm{L}$ & FAPESP \\
\hline Lopes et al. $(2016)^{45}$ & Jan-May 2012 & $\mathrm{DF} / \mathrm{CW}$ & Outpatient & $\begin{array}{l}<75 \mathrm{nmol} / \mathrm{L} \\
50-75 \mathrm{nmol} / \mathrm{L} \\
<50 \mathrm{nmol} / \mathrm{L}\end{array}$ & NR \\
\hline Machado et al. $(2013)^{46}$ & May 2010-Dec 2011 & $\mathrm{SP} / \mathrm{SE}$ & University & $\begin{array}{l}<75 \mathrm{nmol} / \mathrm{L} \\
50-80 \mathrm{nmol} / \mathrm{L} \\
<50 \mathrm{nmol} / \mathrm{L}\end{array}$ & UNIFESP \\
\hline Martins et al. $(2018)^{37}$ & Oct-Dec 2016 & $\mathrm{CE} / \mathrm{NE}$ & \multicolumn{2}{|c|}{$\begin{aligned} \text { Obstetrical care } & <75 \mathrm{nmol} / \mathrm{L} \\
& 50-75 \mathrm{nmol} / \mathrm{L} \\
& <50 \mathrm{nmol} / \mathrm{L}\end{aligned}$} & NR \\
\hline Mendes et al. (2019) ${ }^{47}$ & NR & NR & NR & $25-50 \mathrm{nmol} / \mathrm{L}$ & CNPq \\
\hline Pena et al. $(2015)^{48}$ & Nov 2012-Mar 2013 & $\mathrm{PE} / \mathrm{NE}$ & \multicolumn{2}{|c|}{$\begin{aligned} \text { Obstetrical care } & <75 \mathrm{nmol} / \mathrm{L} \\
& 50-75 \mathrm{nmol} / \mathrm{L} \\
& <50 \mathrm{nmol} / \mathrm{L}\end{aligned}$} & $\mathrm{CNPq}$ \\
\hline $\begin{array}{l}\text { Pereira-Santos }(2014)^{49} \text { and } \\
\text { Pereira-Santos et al. }(2018)^{50}\end{array}$ & & $\mathrm{BA} / \mathrm{NE}$ & \multicolumn{2}{|c|}{$\begin{aligned} \text { Obstetrical care } & <75 \mathrm{nmol} / \mathrm{L} \\
& 50-75 \mathrm{nmol} / \mathrm{L} \\
& <50 \mathrm{nmol} / \mathrm{L}\end{aligned}$} & CNPq and CAPES \\
\hline Peters et al. (2009) $)^{51}$ & Apr-May 2006 & $\mathrm{SP} / \mathrm{SE}$ & $\begin{array}{l}\text { Outpatient/ } \\
\text { Rural }\end{array}$ & $25-75 \mathrm{nmol} / \mathrm{L}$ & FAPESP \\
\hline Santos et al. $(2013)^{52}$ & Apr 2008-Sep 2010 & $\mathrm{PR} / \mathrm{S}$ & School & $\begin{array}{l}<75 \mathrm{nmol} / \mathrm{L} \\
50-75 \mathrm{nmol} / \mathrm{L} \\
<50 \mathrm{nmol} / \mathrm{L}\end{array}$ & CNPq \\
\hline Santos et al. $(2017)^{53}$ & NR & $\mathrm{RS} / \mathrm{S}$ & Outpatient & $\begin{array}{l}<75 \mathrm{nmol} / \mathrm{L} \\
50-75 \mathrm{nmol} / \mathrm{L} \\
<50 \mathrm{nmol} / \mathrm{L}\end{array}$ & CNPq and CAPES \\
\hline Santos et al. $(2019)^{54}$ & 2005-2012 & $\mathrm{RS} / \mathrm{S}$ & NR & $<50 \mathrm{nmol} / \mathrm{L}$ & $\begin{array}{l}\text { CNPq, FAPERGS, and } \\
\text { CAPES }\end{array}$ \\
\hline $\begin{array}{l}\text { Schtscherbyna et al. (2016) } \\
55\end{array}$ & Apr 2008-May 2011 & $\mathrm{RJ} / \mathrm{SE}$ & Outpatient & $<75 \mathrm{nmol} / \mathrm{L}$ & CAPES, FAPERJ, and CNPq \\
\hline Shinjo et al. $(2011)^{56}$ & NR & SP/SE & Outpatient & $<50 \mathrm{nmol} / \mathrm{L}$ & $\begin{array}{l}\text { CNPq and Federico } \\
\text { Foundation }\end{array}$ \\
\hline Simões et al. $(2016)^{57}$ & Apr 2013-Jun 2013 & SP/SE & Obstetrical car & & FAPESP and CAPES \\
\hline
\end{tabular}




\begin{tabular}{|c|c|c|c|c|c|}
\hline Study & Inclusion period & $\begin{array}{l}\text { State/ } \\
\text { region }\end{array}$ & Setting & Cutoff values & Funding \\
\hline & & & & $\begin{array}{l}<75 \mathrm{nmol} / \mathrm{L} \\
50-75 \mathrm{nmol} / \mathrm{L} \\
<50 \mathrm{nmol} / \mathrm{L}\end{array}$ & \\
\hline \multicolumn{6}{|l|}{ Case-control } \\
\hline Dutra et al. $(2019)^{58}$ & Sep 2016-Dec 2017 & SP/SE & Obstetrical & $\begin{array}{l}\mathrm{e}<75 \mathrm{nmol} / \mathrm{L} \\
50-75 \mathrm{nmol} / \mathrm{L} \\
<50 \mathrm{nmol} / \mathrm{L}\end{array}$ & CAPES, CNPq, and FAPESP \\
\hline Menegati et al. (2016) ${ }^{17}$ & 2006-2010 & $\mathrm{RJ} / \mathrm{SE}$ & Outpatient & $\begin{array}{l}<75 \mathrm{nmol} / \mathrm{L} \\
50-75 \mathrm{nmol} / \mathrm{L} \\
<50 \mathrm{nmol} / \mathrm{L}\end{array}$ & CAPES \\
\hline \multicolumn{6}{|l|}{ Prospective cohorts } \\
\hline Benaim et al. $(2019)^{59}$ & Nov 2009-Oct 2011 & $\mathrm{RJ} / \mathrm{SE}$ & Outpatient & $\begin{array}{l}<75 \mathrm{nmol} / \mathrm{L} \\
50-75 \mathrm{nmol} / \mathrm{L} \\
<50 \mathrm{nmol} / \mathrm{L}\end{array}$ & CNPq and FAPERJ \\
\hline $\begin{array}{l}\text { Lepsch et al. }(2017)^{60} \text { and } \\
\text { Figueiredo et al. }(2017 \text {, } \\
2018,2020)^{61-63}\end{array}$ & Nov 2009-Oct 2011 & $\mathrm{RJ} / \mathrm{SE}$ & Obstetrical & $\begin{array}{l}\mathrm{e}<75 \mathrm{nmol} / \mathrm{L} \\
50-75 \mathrm{nmol} / \mathrm{L} \\
<50 \mathrm{nmol} / \mathrm{L}\end{array}$ & CNPq and FAPERJ \\
\hline Medeiros et al. (2016) ${ }^{64}$ & Mar 2010-Jul 2013 & $\mathrm{RJ} / \mathrm{SE}$ & Outpatient & $\begin{array}{l}<75 \mathrm{nmol} / \mathrm{L} \\
50-75 \mathrm{nmol} / \mathrm{L} \\
<50 \mathrm{nmol} / \mathrm{L}\end{array}$ & NR \\
\hline $\begin{array}{l}\text { Weinert et al. }(2014,2016) \\
65,66\end{array}$ & Nov 2009-May 2012 & $\mathrm{RS} / \mathrm{S}$ & Obstetrical & $\begin{array}{l}\mathrm{e}<75 \mathrm{nmol} / \mathrm{L} \\
50-75 \mathrm{nmol} / \mathrm{L} \\
<50 \mathrm{nmol} / \mathrm{L}\end{array}$ & $\begin{array}{l}\text { Hospital de Clínicas de } \\
\text { Porto Alegre }\end{array}$ \\
\hline \multicolumn{6}{|l|}{ Retrospective studies } \\
\hline $\begin{array}{l}\text { Cruz et al. }(2018,2020) \\
18,19,67\end{array}$ & Jan 2011-Jul 2015 & $\mathrm{RJ} / \mathrm{SE}$ & Outpatient & $\begin{array}{l}<75 \mathrm{nmol} / \mathrm{L} \\
50-75 \mathrm{nmol} / \mathrm{L} \\
<50 \mathrm{nmol} / \mathrm{L}\end{array}$ & FAPERJ \\
\hline Rosa et al. $(2013)^{68}$ & NR & $\mathrm{RJ} / \mathrm{SE}$ & NR & $38-225 \mathrm{nmol} / \mathrm{L}$ & NR \\
\hline
\end{tabular}

Abbreviations: NR, not reported; SD, standard deviation.

State/Region: BA, Bahia; CE, Ceará; CW, Center-west; DF, Distrito Federal; MA, Maranhão; MG, Minas Gerais; NE, Northeast; PB, Paraíba; PE, Pernambuco; PR, Paraná; RJ, Rio de Janeiro; RS, Rio Grande do Sul; S, South; SC, Santa Catarina; SE, Southeast; SP, São Paulo. Funding/Institutions: CAPES, Coordenação de Aperfeiçoamento de Pessoal de Nível Superior; CNPq, Conselho Nacional de Desenvolvimento Científico e Tecnológico; FAPEMIG, Fundação de Amparo à Pesquisa do Estado de Minas Gerais; FAPERGS, Fundação de Amparo à Pesquisa do Estado do Rio Grande do Sul; FAPERJ, Fundação de Amparo à Pesquisa do Estado do Rio de Janeiro; FAPESP, Fundação de Amparo à Pesquisa do Estado de São Paulo; UEL, Universidade Estadual de Londrina; UNIFESP, Universidade Federal de São Paulo.

deficiencies. The detailed assessment of the methodological quality of the included studies is presented in Chart 3. ${ }^{3,17-19,32-35,37-66}$

Most studies $(n=26)$ used common cutoff values (vitamin D deficiency: $<50 \mathrm{nmol} / \mathrm{L}$ or $<20 \mathrm{ng} / \mathrm{mL}$; vitamin D insufficiency: $50-75 \mathrm{nmol} / \mathrm{L}$ or $20-30 \mathrm{ng} / \mathrm{mL}$; and vitamin $\mathrm{D}$ deficiency or insufficiency: $<75 \mathrm{nmol} / \mathrm{L}$ or $30 \mathrm{ng} / \mathrm{mL}$ ) (Chart 1) and were included in meta-analyses.

The prevalence of vitamin D deficiency ranged from 3 to $85 \%$, insufficiency from $15 \%$ to $68 \%$, and deficiency or insufficiency from 34 to $94 \%$. In the meta-analysis for the basecase, an overall prevalence of vitamin D deficiency of $35 \%$ (95\%CI: $34-37 \%$ ), insufficiency of $42 \%$ (95\%CI: $41-44 \%$ ) (-Fig. 1), and deficiency or insufficiency of $72 \%(95 \% \mathrm{CI}$ : 71-74\%) (-Appendix A, supplementary material) were obtained. ${ }^{23}$ When the population subgroups were considered, lower and higher prevalence of vitamin D deficiency were identified in pregnant (27\%) and postpartum women (48\%), respectively; and lower and higher prevalence of vitamin D insufficiency were associated with adolescents (37\%) and women of childbearing age (50\%) (-Fig. 1).

Some studies reported subgroup analyses: higher deficiency or insufficiency prevalence values were found in adolescence $(p$ value $=0.02),{ }^{40}$ first pregnancy $(p=0.01),{ }^{40} \geq 11$ years of schooling $(p=0.03),{ }^{49,50}$ first gestational trimester $(p=0.01),{ }^{49,50}$ face and hands exposed to the sun $(p=0.01),{ }^{49,50}$ methods of commuting by motor vehicles $(p=0.01),{ }^{49,50}$ and winter $(p<0.001){ }^{49,50,60-63}$ Except for gestational trimester, no metaanalyses for these subgroups were possible due to the small number of studies in each subgroup, or different categorization for the same subgroup. Four studies assessed vitamin D status throughout gestational trimesters, with little variation among trimesters of vitamin D deficiency (15-20\%) or insufficiency (3449\%) and wide confidence intervals (-Fig. 2 ).

Five studies assessed vitamin D in women post-RYGB, and two of them analyzed pregnant women after RYGB. No metaanalysis was possible due to the different cutoff values and categories used. The deficiency, insufficiency, and deficiency 
414 Deficiency and Insufficiency of Vitamin D in Women of Childbearing Age Lucchetta et al.

Chart 2 Description of the characteristics of the included participants

\begin{tabular}{|c|c|c|c|c|c|c|}
\hline Study & $\begin{array}{l}\text { Main } \\
\text { characteristic (N) }\end{array}$ & Ethnicity & Comorbidities & $\begin{array}{l}\text { Medicine/ } \\
\text { supplement }\end{array}$ & $\begin{array}{l}\text { Body mass } \\
\text { index, } \mathrm{kg} / \mathrm{m}^{2}\end{array}$ & Mean age, years \\
\hline $\begin{array}{l}\text { Araújo et al. (2017), } \\
\text { Queiroz (2016), }{ }^{33} \text { and } \\
\text { Queiroz et al. (2019) })^{34}\end{array}$ & Adolescents (136) & Brown (62\%) & $\begin{array}{l}\text { NR (Excluded some } \\
\text { conditions) }\end{array}$ & NR/None & $\begin{array}{l}\text { Normal } \\
\text { weight (72\%) }\end{array}$ & $17\left( \pm\right.$ SD 1) ${ }^{b}$ \\
\hline Benaim et al. $(2019)^{59}$ & $\begin{array}{l}\text { Pregnant women } \\
\text { (181) }\end{array}$ & Mixed (47\%) & $\begin{array}{l}\text { NR (Excluded some } \\
\text { conditions) }\end{array}$ & NR/Yes & $\begin{array}{l}\text { Median: } 24 \\
\text { (IQR 22-27) }\end{array}$ & $\begin{array}{l}\text { Median: } 26 \\
\text { (IQR 22-31) }\end{array}$ \\
\hline Chrisostomo et al. $(2018)^{38}$ & $\begin{array}{l}\text { Pregnant women } \\
(520)\end{array}$ & $\begin{array}{l}\text { Euro-descendant } \\
(52 \%)\end{array}$ & $\begin{array}{l}\text { Preeclampsia; GDM; } \\
\text { HIV+ }\end{array}$ & $\begin{array}{l}\text { Antiretroviral/ } \\
\text { None }\end{array}$ & $\begin{array}{l}\text { Median } 31 \\
\text { (IQR: } 27 ; 35 \text { ) }\end{array}$ & $\begin{array}{l}\text { Median: } 30 \\
\text { (IQR: 25-35) }\end{array}$ \\
\hline $\begin{array}{l}\text { Cruz et al. } \\
(2018,2020)^{18,19,67}\end{array}$ & $\begin{array}{l}\text { Pregnant and } \\
\text { nonpregnant } \\
\text { women (121) }\end{array}$ & NR & $\begin{array}{l}\text { RYGB (Excluded some } \\
\text { conditions) }\end{array}$ & NR/Yes e & $\begin{array}{l}43( \pm \text { SD } 3) \text { to } \\
44( \pm \text { SD } 6)\end{array}$ & $\begin{array}{l}30( \pm S D 4) \text { to } \\
32( \pm S D 4)\end{array}$ \\
\hline Rosa et al. $(2013)^{68}$ & $\begin{array}{l}\text { Women of } \\
\text { childbearing } \\
\text { age }(56)\end{array}$ & NR & RYGB & NR/Yes ${ }^{f}$ & $46( \pm S D 8)$ & $35( \pm S D$ 9) \\
\hline $\begin{array}{l}\text { Duran de Campos et al. } \\
(2008)^{39}\end{array}$ & $\begin{array}{l}\text { Women of } \\
\text { childbearing } \\
\text { age }(30)\end{array}$ & $\begin{array}{l}\text { NR (excluded } \\
\text { nonwhite) }\end{array}$ & RYGB & NR & $\begin{array}{l}29( \pm S D 2.3) \text { to } \\
47( \pm S D 8.6)\end{array}$ & $46( \pm S D 3)$ \\
\hline de Oliveira et al. $(2020)^{35}$ & Adolescents (100) & Nonwhite (54\%) & NR & NR & $\begin{array}{l}\text { Normal weight } \\
(71 \%)\end{array}$ & $15-17$ (59\%) \\
\hline Souza et al. (2019) & $\begin{array}{l}\text { Pregnant women } \\
\text { (71) }\end{array}$ & Dart (62\%) & Healthy & NR/None & NR & $26( \pm S D 6)$ \\
\hline Delmonico et al. $(2018)^{41}$ & $\begin{array}{l}\text { Women of } \\
\text { childbearing } \\
\text { age }(20)\end{array}$ & NR & $\begin{array}{l}\text { Malignant breast } \\
\text { lesions }\end{array}$ & NR & NR & 37 \\
\hline Prado et al. $(2015)^{3}$ & $\begin{array}{l}\text { Postpartum } \\
\text { women (226) }\end{array}$ & White (52\%) & NR & NR/Yes (97\%) & NR & 28 (range 20-44) \\
\hline Dutra et al. (2019) & $\begin{array}{l}\text { Postpartum } \\
\text { women }(126)^{g}\end{array}$ & NR & Hypertension (23\%) & NR/Yes & $\begin{array}{l}26( \pm \text { SD 6) to } \\
27( \pm S D 5)\end{array}$ & $\begin{array}{l}25( \pm \text { SD 7) to } \\
26( \pm \text { SD 7) }\end{array}$ \\
\hline Ferreira et al. $(2015)^{42}$ & $\begin{array}{l}\text { Women of } \\
\text { childbearing } \\
\text { age }(73)\end{array}$ & White (68\%) & $\begin{array}{l}\text { NR (Excluded some } \\
\text { conditions) }\end{array}$ & NR/None & $26( \pm S D 1)$ & $32( \pm$ SD 1) \\
\hline Flauzino et al. $(2017)^{43}$ & $\begin{array}{l}\text { Women of } \\
\text { childbearing } \\
\text { age (205) }\end{array}$ & $\begin{array}{l}\text { Caucasian } \\
(71-78 \%)^{b}\end{array}$ & $\mathrm{HIV}+$ & $\begin{array}{l}\text { Antiretroviral/ } \\
\text { None }\end{array}$ & $\begin{array}{l}25( \pm \text { SD 0) to } \\
26( \pm \text { SD 0 })^{b}\end{array}$ & $40( \pm S D 1)^{b}$ \\
\hline $\begin{array}{l}\text { Lepsch et al. }(2017)^{60} \text { and } \\
\text { Figueiredo et al. } \\
(2017,2018,2020)^{61-63}\end{array}$ & $\begin{array}{l}\text { Pregnant women } \\
\text { (199) }\end{array}$ & Mixed (46\%) & $\begin{array}{l}\text { NR (Excluded some } \\
\text { conditions) }\end{array}$ & None/None & $<25(60 \%)$ & $27( \pm S D 6)$ \\
\hline Lopes et al. $(2015)^{44}$ & Adolescents (97) & NR & NR & NR & $26( \pm S D 9)$ & $16( \pm S D 1)$ \\
\hline Lopes et al. $(2016)^{45}$ & $\begin{array}{l}\text { Women of } \\
\text { childbearing } \\
\text { age (369) }\end{array}$ & NR & Infertility and control & NR/None & NR & $\begin{array}{l}36( \pm \text { SD } 4) \text { to } \\
37( \pm S D 4)\end{array}$ \\
\hline Machado et al. $(2013)^{46}$ & $\begin{array}{l}\text { Pregnant women } \\
\text { (49) }\end{array}$ & NR & $\mathrm{HIV}+$ & $\begin{array}{l}\text { Antiretroviral/ } \\
\text { None }\end{array}$ & $\begin{array}{l}\text { Excessive } \\
\text { gestational } \\
\text { weight (35\%) }\end{array}$ & $30( \pm S D 7)$ \\
\hline Martins et al. $(2018)^{37}$ & $\begin{array}{l}\text { Postpartum } \\
\text { women (225) }\end{array}$ & Dark (79\%) & $\begin{array}{l}\text { Urinary tract } \\
\text { infection (32\%), } \\
\text { hypertension (9\%), } \\
\text { GDM (1\%), and } \\
\text { bleeding ( } 8 \%)\end{array}$ & NR/Yes (64\%) & $\begin{array}{l}\text { Overweight or } \\
\text { obesity (34\%) }\end{array}$ & $26( \pm S D 7)$ \\
\hline Medeiros et al. $(2016)^{64}$ & $\begin{array}{l}\text { Pregnant women } \\
\text { (46) }\end{array}$ & NR & RYGB & NR/Yes ${ }^{i}$ & 28 to $44( \pm S D 6)$ & $31( \pm S D 5)$ \\
\hline Mendes et al. (2019) ${ }^{47}$ & $\begin{array}{l}\text { Women of } \\
\text { childbearing } \\
\text { age (79) }\end{array}$ & White $(63 \%)$ & NR & NR/None & $24( \pm S D 5)$ & $\begin{array}{l}\text { Median: } 27 \\
\text { (IQR 24-31) }\end{array}$ \\
\hline Menegati et al. (2016) ${ }^{17}$ & $\begin{array}{l}\text { Women of } \\
\text { childbearing } \\
\text { age (58) }\end{array}$ & NR & $\begin{array}{l}\text { RYGB and control } \\
\text { (obesity) (Excluded } \\
\text { some conditions) }^{\mathrm{j}}\end{array}$ & $\begin{array}{l}\text { NR/Yes } \\
\text { (calcium) }\end{array}$ & $\begin{array}{l}35(\mathrm{Cl} 95 \% 33-37) \\
\text { to } 52(\mathrm{Cl} 95 \% \\
40-73)\end{array}$ & $\begin{array}{l}39(\mathrm{Cl} 95 \% 36-42) \\
\text { to } 40(\mathrm{Cl} 95 \% \\
38-42)\end{array}$ \\
\hline Pena et al. $(2015)^{48}$ & & Nonwhite (82\%) & & NR & IQR: 21-37 & IQR: 19-33 \\
\hline
\end{tabular}




\begin{tabular}{|c|c|c|c|c|c|c|}
\hline Study & $\begin{array}{l}\text { Main } \\
\text { characteristic (N) }\end{array}$ & Ethnicity & Comorbidities & $\begin{array}{l}\text { Medicine/ } \\
\text { supplement }\end{array}$ & $\begin{array}{l}\text { Body mass } \\
\text { index, } \mathrm{kg} / \mathrm{m}^{2}\end{array}$ & Mean age, years \\
\hline & $\begin{array}{l}\text { Pregnant and } \\
\text { nonpregnant (179) }\end{array}$ & & $\begin{array}{l}\text { Preeclampsia and } \\
\text { gestational obesity }\end{array}$ & & & \\
\hline $\begin{array}{l}\text { Pereira-Santos }(2014)^{49} \\
\text { and Pereira-Santos et al. } \\
(2018)^{50}\end{array}$ & $\begin{array}{l}\text { Pregnant women } \\
(190)\end{array}$ & Nonblack (68\%) & $\begin{array}{l}\text { NR (Excluded some } \\
\text { conditions) }\end{array}$ & NR/Yes (5\%) & Overweight (43\%) & $18-29(63 \%)$ \\
\hline Peters et al. $(2009)^{51}$ & Adolescents (71) & $\begin{array}{l}\text { NR (excluded } \\
\text { nonwhite) }\end{array}$ & $\begin{array}{l}\text { NR (Excluded some } \\
\text { conditions) }\end{array}$ & NR & $22( \pm S D 0)$ & $18( \pm S D$ 1) \\
\hline Santos et al. $(2013)^{52}$ & Adolescents (198) & NR & Healthy & NR/None & $\begin{array}{l}\text { Normal weight } \\
(76 \%)\end{array}$ & $13( \pm S D 2)$ \\
\hline Santos et al. $(2017)^{53}$ & $\begin{array}{l}\text { Women of } \\
\text { childbearing } \\
\text { age (102) }\end{array}$ & White (94\%) & $\begin{array}{l}\text { Polycystic ovary } \\
\text { syndrome and } \\
\text { controls }\end{array}$ & NR & $\begin{array}{l}27( \pm S D 6) \text { to } \\
30( \pm S D 6)\end{array}$ & $\begin{array}{l}23( \pm \text { SD 7) to } \\
25( \pm \text { SD 8) }\end{array}$ \\
\hline Santos et al. (2019) ${ }^{54}$ & $\begin{array}{l}\text { Women of } \\
\text { childbearing } \\
\text { age (61) }\end{array}$ & Caucasian (80\%) & Healthy & $\begin{array}{l}\text { NR/Yes (calcium } \\
\text { and vitamin D) }\end{array}$ & $29( \pm S D 8)$ & $37( \pm S D 11)$ \\
\hline $\begin{array}{l}\text { Schtscherbyna et al. } \\
(2016)^{55}\end{array}$ & $\begin{array}{l}\text { Adolescents and } \\
\text { young adults ( } 35 \text { ) }\end{array}$ & White $(35 \%)^{b}$ & $\mathrm{HIV}+$ & $\begin{array}{l}\text { Antiretroviral/ } \\
\text { NR }\end{array}$ & Normal $(62 \%)^{b}$ & $\begin{array}{l}\text { Around } 18 \\
( \pm S D 2)^{b}\end{array}$ \\
\hline Shinjo et al. $(2011)^{56}$ & $\begin{array}{l}\text { Women of } \\
\text { childbearing } \\
\text { age }(20)\end{array}$ & White (75\%) & $\begin{array}{l}\text { Juvenile onset of } \\
\text { systemic sclerosis } \\
\text { and controls }\end{array}$ & NR & NR & $\begin{array}{l}21( \pm S D 2) \text { to } \\
21( \pm S D 2)\end{array}$ \\
\hline Simões et al. $(2016)^{57}$ & $\begin{array}{l}\text { Postpartum } \\
\text { women (99) }\end{array}$ & $\begin{array}{l}\text { Blacks or } \\
\text { mulatto (58\%) }\end{array}$ & $\begin{array}{l}\text { NR (Excluded some } \\
\text { conditions) }\end{array}$ & NR/Yes (9\%) & $\begin{array}{l}\text { Overweight } \\
\text { or obese }(69 \%)\end{array}$ & $26( \pm S D 5)$ \\
\hline $\begin{array}{l}\text { Weinert et al. } \\
(2014,2016)^{65,66}\end{array}$ & $\begin{array}{l}\text { Pregnant women } \\
\text { (184) }\end{array}$ & White (74\%) & $\begin{array}{l}\text { GDM }(100 \%) ; \\
\text { Hypertension (22\%) }\end{array}$ & NR/None & $\begin{array}{l}27( \pm \text { SD 5) to } \\
30( \pm \text { SD 7) }\end{array}$ & $32( \pm S D 6)$ \\
\hline
\end{tabular}

Abbreviations: $\mathrm{Cl}$, confidence interval; GDM, gestational diabetes mellitus; HIV, human immunodeficiency virus; IQR, interquartile range; NR, not reported; RYGB, Roux-en-Y gastric bypass; SD, standard deviation. Notes: A - Pregnant, breastfed, carriers of chronic diseases (diabetes, hypertension, chronic kidney disease), chronic alcoholics, and chronic smokers were excluded. B - Both genders. C-Without any known infectious or chronic noncommunicable diseases (except obesity). D - Disabsorptive and restrictive surgeries prior to RYGB, disabsorptive syndromes, cancer and liver and/or kidney diseases (except hepatic steatosis), hypolipidemic or hypoglycemic use, active thyroid disorders, metabolic bone diseases, chronic use of diuretics or calcium channel blockers, female smokers, and presence of gestational diabetes were excluded. E - 850 mg of calcium carbonate and $600 \mathrm{IU}$ of vitamin D3; when inadequacy of vitamin D was found in the preoperative period, all participants consumed $1500 \mathrm{IU}$ of vitamin D; in addition, in case of pregnancy after RYGB, supplementation was adjusted from 1500 to $2000 \mathrm{IU}$ vitamin D and 1200 mg after the immediate confirmation. F - Daily dietary supplementation of $500 \mathrm{mg}$ of calcium carbonate and $400 \mathrm{Ul}$ of vitamin D for an undetermined length of time. G - Mothers' full-term births, and mothers' preterm births. H - Women with smoking; eating disorders; major depression; any metabolic disease, such as diabetes mellitus or hypothyroidism; any chronic diseases severely affecting the CV, gastrointestinal, and renal systems; and pregnancy or lactation were excluded; I - $850 \mathrm{mg}$ of calcium carbonate and $600 \mathrm{IU}$ of vitamin D3. J - Women with malignant tumors or infectious diseases; were postmenopausal; were taking drugs that affect bone metabolism (bisphosphonates, estrogens, anticonvulsants, glucocorticoids); were pregnant; had malabsorption syndrome, primary hyperparathyroidism, renal, or liver failure; or weighed $>120 \mathrm{~kg}$ were excluded. $\mathrm{K}$ - Women with multiple pregnancies, preeclampsia, kidney problems, HIV and women who had not fasted for the blood collection were excluded. L - Chronic illness, pregnancy, and obesity were excluded. M - Alcohol use, hyperglycemia, hypertension, preterm/post-term deliveries and adolescent pregnancy, were excluded.

or insufficiency ranged from 12 to $39 \%, 41$ to $54 \%$, and 60 to $91 \%$, respectively.

Cumulative meta-analyses were performed considering the year of publication, showing a trend toward a lower prevalence of vitamin D deficiency, and higher prevalence of vitamin D insufficiency and vitamin D deficiency or insufficiency, with a slight join point in 2017 (-Appendix B, supplementary material). ${ }^{23}$ Meta-regression analyses were conducted for publication year, and a moderator effect was not identified $(p>0.05)$ (-Appendix $C$, supplementary material). ${ }^{23}$ Meta-regression or subgroup analyses for other variables were not possible, and neither were cumulative meta-analyses regarding gestational trimesters, because the minimum number of studies required was not met.

Sensitivity analyses by the leave-one-out method were not able to reduce heterogeneity (93-96\%) and the overall preva- lence ranged from 32 to $37 \%$ for vitamin D deficiency, 41 to $44 \%$ for vitamin D insufficiency, and 71 to $73 \%$ for vitamin D deficiency or insufficiency (-Appendix D, supplementary material). ${ }^{23}$ The study with more influence in the variations was Prado et al., ${ }^{3}$ conducted in Minas Gerais, in 2012, with postpartum women taking supplements (97\%). Sensitivity analyses with alternative statistical methods identified values of prevalence ranging from 35 to $37 \%$ for vitamin D deficiency, 41 to $43 \%$ for vitamin D insufficiency, and 69 to $72 \%$ for vitamin D deficiency or insufficiency (-Appendix A, supplementary material). ${ }^{23}$ It was not possible to conduct sensitivity analyses regarding gestational trimesters.

Potential publication biases were not identified in vitamin D deficiency ( $p=0.84)$, insufficiency $(p=0.60)$, or deficiency or insufficiency $(p=0.54)$ in statistical or visual analyses (-Appendix E, supplementary material). ${ }^{23}$ It was also not 
Chart 3 Methodological and reporting quality assessment, considering the Joanna Briggs Institute tool for prevalence studies

\begin{tabular}{|c|c|c|c|c|c|c|c|c|c|}
\hline \multirow[b]{2}{*}{ Study } & \multicolumn{9}{|c|}{ Question } \\
\hline & 1 & 2 & 3 & 4 & 5 & 6 & 7 & 8 & 9 \\
\hline $\begin{array}{l}\text { Araújo et al. (2017), }{ }^{32} \text { Queiroz (2016), } \\
\text { and Queiroz et al. (2019) }\end{array}$ & Yes & Yes & $\mathrm{No}^{\mathrm{c}}$ & Yes & Yes & $\mathrm{N} / \mathrm{A}$ & Yes & Yes & $\mathrm{No}^{\mathrm{h}}$ \\
\hline Benaim et al. $(2019)^{59}$ & Yes & $\mathrm{No}^{\mathrm{a}}$ & $\mathrm{No}^{\mathrm{d}}$ & $\mathrm{No}^{\mathrm{e}}$ & Unclear & $\mathrm{N} / \mathrm{A}$ & Yes & Yes & Unclear ${ }^{\mathrm{i}}$ \\
\hline Chrisostomo et al. $(2018)^{38}$ & Yes & $\mathrm{No}^{\mathrm{a}}$ & $\mathrm{No}^{\mathrm{d}}$ & Yes & Yes & $N / A$ & Yes & Yes & Yes $^{\mathrm{j}}$ \\
\hline Cruz et al. $(2018,2020)^{18,19,67}$ & Yes & $\mathrm{No}^{\mathrm{a}}$ & $\mathrm{No}^{\mathrm{d}}$ & $\mathrm{No}^{\mathrm{e}}$ & Unclear & $\mathrm{N} / \mathrm{A}$ & Yes & $\mathrm{No}^{\mathrm{g}}$ & $\mathrm{No}^{\mathrm{h}}$ \\
\hline Rosa et al. $(2013)^{68}$ & Yes & $\mathrm{No}^{\mathrm{a}}$ & $\mathrm{No}^{\mathrm{d}}$ & $\mathrm{No}^{\mathrm{e}}$ & Unclear & $\mathrm{N} / \mathrm{A}$ & $\mathrm{No}^{f}$ & $\mathrm{No}^{\mathrm{g}}$ & $\mathrm{No}^{\mathrm{h}}$ \\
\hline Duran de Campos et al. $(2008)^{39}$ & Yes & $\mathrm{No}^{\mathrm{a}}$ & $\mathrm{No}^{\mathrm{d}}$ & $\mathrm{No}^{\mathrm{e}}$ & Unclear & $\mathrm{N} / \mathrm{A}$ & $\mathrm{No}^{f}$ & Yes & $\mathrm{No}^{\mathrm{h}}$ \\
\hline de Oliveira et al. $(2020)^{35}$ & Yes & Yes & Yes & $\mathrm{No}^{\mathrm{e}}$ & Unclear & $N / A$ & Yes & Yes & $\mathrm{No}^{\mathrm{h}}$ \\
\hline Souza et al. (2019) ${ }^{40}$ & Yes & $\mathrm{No}^{\mathrm{a}}$ & $\mathrm{No}^{\mathrm{d}}$ & $\mathrm{No}^{\mathrm{e}}$ & Unclear & $\mathrm{N} / \mathrm{A}$ & Yes & Yes & $\mathrm{No}^{\mathrm{h}}$ \\
\hline Delmonico et al. $(2018)^{41}$ & Yes & $\mathrm{No}^{\mathrm{a}}$ & $\mathrm{No}^{\mathrm{c}}$ & $\mathrm{No}^{\mathrm{e}}$ & Unclear & $\mathrm{N} / \mathrm{A}$ & Yes & Yes & $\mathrm{No}^{\mathrm{h}}$ \\
\hline Prado et al. $(2015)^{3}$ & Yes & $\mathrm{No}^{\mathrm{a}}$ & $\mathrm{No}^{\mathrm{d}}$ & $\mathrm{No}^{\mathrm{e}}$ & Unclear & $N / A$ & Yes & Yes & Unclear $^{\mathrm{i}}$ \\
\hline Dutra et al. $(2019)^{58}$ & Yes & $\mathrm{No}^{\mathrm{a}}$ & $\mathrm{No}^{\mathrm{d}}$ & $\mathrm{No}^{\mathrm{e}}$ & Unclear & $N / A$ & Yes & $\mathrm{No}^{\mathrm{g}}$ & $\mathrm{No}^{\mathrm{h}}$ \\
\hline Ferreira et al. $(2015)^{42}$ & Yes & $\mathrm{No}^{\mathrm{a}}$ & $\mathrm{No}^{\mathrm{d}}$ & $\mathrm{No}^{\mathrm{e}}$ & Unclear & $\mathrm{N} / \mathrm{A}$ & Yes & Yes & $\mathrm{No}^{\mathrm{h}}$ \\
\hline Flauzino et al. $(2017)^{43}$ & Yes & $\mathrm{No}^{\mathrm{a}}$ & $\mathrm{No}^{\mathrm{d}}$ & Yes & Yes & $\mathrm{N} / \mathrm{A}$ & Yes & $\mathrm{No}^{\mathrm{g}}$ & Unclear $^{\mathrm{i}}$ \\
\hline $\begin{array}{l}\text { Lepsch et al. }(2017)^{60} \text { and } \\
\text { Figueiredo et al. }(2017,2018,2020)^{61-63}\end{array}$ & Yes & $\mathrm{No}^{\mathrm{a}}$ & $\mathrm{No}^{\mathrm{d}}$ & Yes & Yes & $N / A$ & Yes & $\mathrm{No}^{\mathrm{g}}$ & Unclear $^{\mathrm{i}}$ \\
\hline Lopes et al. $(2015)^{44}$ & Yes & $\mathrm{No}^{\mathrm{a}}$ & $\mathrm{No}^{\mathrm{d}}$ & $\mathrm{No}^{\mathrm{e}}$ & Unclear & $\mathrm{N} / \mathrm{A}$ & Yes & Yes & $\mathrm{No}^{\mathrm{h}}$ \\
\hline Lopes et al. $(2016)^{45}$ & Yes & $\mathrm{No}^{\mathrm{a}}$ & $\mathrm{No}^{\mathrm{d}}$ & $\mathrm{No}^{\mathrm{e}}$ & Unclear & $\mathrm{N} / \mathrm{A}$ & Yes & Yes & Yes $^{\mathrm{j}}$ \\
\hline Machado et al. $(2013)^{46}$ & Yes & $\mathrm{No}^{\mathrm{a}}$ & $\mathrm{No}^{\mathrm{d}}$ & Yes & Yes & $\mathrm{N} / \mathrm{A}$ & Yes & Yes & $\mathrm{No}^{\mathrm{h}}$ \\
\hline Martins et al. $(2018)^{37}$ & Yes & $\mathrm{No}^{\mathrm{b}}$ & $\mathrm{No}^{\mathrm{d}}$ & Yes & Yes & $\mathrm{N} / \mathrm{A}$ & Yes & Yes & Unclear $^{\mathrm{i}}$ \\
\hline Medeiros et al. $(2016)^{64}$ & Yes & $\mathrm{No}^{\mathrm{a}}$ & $\mathrm{No}^{\mathrm{d}}$ & $\mathrm{No}^{\mathrm{e}}$ & Unclear & $\mathrm{N} / \mathrm{A}$ & Yes & Yes & $\mathrm{No}^{\mathrm{h}}$ \\
\hline Mendes et al. (2019) ${ }^{47}$ & Yes & $\mathrm{No}^{\mathrm{a}}$ & $\mathrm{No}^{\mathrm{d}}$ & $\mathrm{No}^{\mathrm{e}}$ & Unclear & $N / A$ & $\mathrm{No}^{f}$ & Yes & $\mathrm{No}^{\mathrm{h}}$ \\
\hline Menegati et al. $(2016)^{17}$ & Yes & $\mathrm{No}^{\mathrm{a}}$ & $\mathrm{No}^{\mathrm{d}}$ & $\mathrm{No}^{\mathrm{e}}$ & Unclear & $\mathrm{N} / \mathrm{A}$ & Yes & Yes & $\mathrm{No}^{\mathrm{h}}$ \\
\hline Pena et al. $(2015)^{48}$ & Yes & $\mathrm{No}^{\mathrm{a}}$ & $\mathrm{No}^{\mathrm{d}}$ & Yes & Yes & $\mathrm{N} / \mathrm{A}$ & Yes & Yes & $\mathrm{No}^{\mathrm{h}}$ \\
\hline $\begin{array}{l}\text { Pereira-Santos }(2014)^{49} \text { and } \\
\text { Pereira-Santos et al. }(2018)^{50}\end{array}$ & Yes & $\mathrm{No}^{\mathrm{a}}$ & $\mathrm{No}^{\mathrm{d}}$ & $\mathrm{No}^{\mathrm{e}}$ & Unclear & $N / A$ & Yes & Yes & Unclear $^{\mathrm{i}}$ \\
\hline Peters et al. $(2009)^{51}$ & Yes & $\mathrm{No}^{\mathrm{a}}$ & $\mathrm{No}^{\mathrm{d}}$ & $\mathrm{No}^{\mathrm{e}}$ & Unclear & $\mathrm{N} / \mathrm{A}$ & $\mathrm{No}^{f}$ & $\mathrm{No}^{\mathrm{g}}$ & $\mathrm{No}^{\mathrm{h}}$ \\
\hline Santos et al. $(2013)^{52}$ & Yes & $\mathrm{No}^{\mathrm{a}}$ & $\mathrm{No}^{\mathrm{c}}$ & $\mathrm{No}^{\mathrm{e}}$ & Unclear & $\mathrm{N} / \mathrm{A}$ & Yes & $\mathrm{No}^{\mathrm{g}}$ & Unclear $^{\mathrm{i}}$ \\
\hline Santos et al. $(2017)^{53}$ & Yes & $\mathrm{No}^{\mathrm{a}}$ & $\mathrm{No}^{\mathrm{d}}$ & Yes & Yes & $N / A$ & Yes & $\mathrm{No}^{\mathrm{g}}$ & $\mathrm{No}^{\mathrm{h}}$ \\
\hline Santos et al. $(2019)^{54}$ & Yes & $\mathrm{No}^{\mathrm{a}}$ & $\mathrm{No}^{\mathrm{d}}$ & Yes & Yes & $\mathrm{N} / \mathrm{A}$ & Yes & Yes & $\mathrm{No}^{\mathrm{h}}$ \\
\hline Schtscherbyna et al. $(2016)^{55}$ & Yes & $\mathrm{No}^{\mathrm{a}}$ & $\mathrm{No}^{\mathrm{d}}$ & Yes & Yes & $\mathrm{N} / \mathrm{A}$ & Yes & Yes & $\mathrm{No}^{\mathrm{h}}$ \\
\hline Shinjo et al. $(2011)^{56}$ & Yes & $\mathrm{No}^{\mathrm{a}}$ & $\mathrm{No}^{\mathrm{d}}$ & $\mathrm{No}^{\mathrm{e}}$ & Unclear & $\mathrm{N} / \mathrm{A}$ & Yes & Yes & $\mathrm{No}^{\mathrm{h}}$ \\
\hline Simões et al. $(2016)^{57}$ & Yes & $\mathrm{No}^{\mathrm{a}}$ & $\mathrm{No}^{\mathrm{d}}$ & $\mathrm{No}^{\mathrm{e}}$ & Unclear & $\mathrm{N} / \mathrm{A}$ & Yes & Yes & $\mathrm{No}^{\mathrm{h}}$ \\
\hline Weinert et al. $(2014,2016)^{65,66}$ & Yes & $\mathrm{No}^{\mathrm{a}}$ & $\mathrm{No}^{\mathrm{d}}$ & Yes & Yes & $N / A$ & Yes & Yes & Unclear $^{\mathrm{i}}$ \\
\hline
\end{tabular}

Abbreviation: N/A, not applicable. Notes: 1. Was the sample frame appropriate to address the target population? 2. Were study participants recruited in an appropriate way? 3 . Was the sample size adequate? 4 . Were the study's subjects and setting described in detail? 5 . Was data analysis conducted with sufficient coverage of the identified sample? 6 . Were valid methods used for the identification of the condition? 7 . Was the condition measured in a standard, reliable way for all participants? 8. Was there appropriate statistical analysis? 9. Was the response rate adequate, and if not, was the low response rate managed appropriately? a - Not reported, convenience sampling was considered. b - Reported convenience sampling. $\mathbf{c}-$ The target sample size reported was low. $\mathbf{d}$ - A target sample size was not reported. e - Did not report at least two of the following information: ethnicity, comorbidities, medicines/supplementation, body mass index, age, educational level, or income per capita. $\mathbf{f}-\mathrm{Vitamin} \mathrm{D}$ cutoff different than usual. $\mathbf{g}$ - Not reported numerator ( $\mathrm{n}$ ) or denominator $(\mathrm{N})$ of prevalence). $\mathbf{h}$ - Not reported numerator (n) or denominator $(\mathrm{N})$ of prevalence. $\mathbf{i}-$ The studies presented a response rate below 176 participants to vitamin $\mathrm{D}$ assessment. $\mathrm{j}$ - The studies presented a response rate between 176 and 345 (vitamin D), therefore is unclear if the sample size is appropriate, since a reliable estimate was not possible. $\mathbf{k}$ - The studies presented a response rate higher than 345, therefore, high confidence about good response was achieved. 
possible to conduct statistical and visual analyses of publication bias for meta-analysis along gestational trimesters.

Four studies reported different cutoff values and were not included in any meta-analysis. They identified prevalence values ranging from $11 \%$ to $75 \%$ : Duran de Campos et al. $^{39}$ identified serum 25(OH)D levels between 12.5 and 25 $\mathrm{nmol} / \mathrm{L}(5-10 \mathrm{ng} / \mathrm{mL})$ in $50 \%$ of the participants, and between 25 and $50 \mathrm{nmol} / \mathrm{L}(10-20 \mathrm{ng} / \mathrm{mL})$ in $40 \%$ of the participants; Mendes et al. ${ }^{47}$ identified $11 \%$ of the participants with values between 25 and $50 \mathrm{nmol} / \mathrm{L}$; Peters et al. ${ }^{51}$ identified $61 \%$ of the participants with values between 25 and $75 \mathrm{nmol} / \mathrm{L}$ (11$30 \mathrm{ng} / \mathrm{mL}$ ); and Rosa et al. ${ }^{68}$ identified $55 \%$ and $75 \%$ of the participants with values between 15 and $90 \mathrm{ng} / \mathrm{mL}$ in preand postoperative RYGB, respectively.

\section{Discussion}

In this systematic review, 31 studies assessing prevalence of inadequate levels of vitamin $\mathrm{D}$ in women of childbearing age were found, reporting vitamin D deficiency (3-85\%), insufficiency (15-68\%), and deficiency or insufficiency (34-94\%), with a mean prevalence of $35 \%, 42 \%$, and $72 \%$ identified through the meta-analysis, respectively.

Redundant evidence of vitamin D levels was identified, especially for women of childbearing age in Brazil, to the detriment of population subgroups such as pregnant women, women who have recently given birth, and adolescents. In 2019, Pereira-Santos et al. ${ }^{4}$ identified 72 studies that reported prevalence of vitamin D deficiency (28\%) and insufficiency (45\%) in the general population, and five studies that reported prevalence of $33 \%$ and $49 \%$, respectively, in pregnant women. Although our systematic review identified the double of studies in pregnant women and 22 studies with women of childbearing age, our prevalence is similar to the Pereira-Santos' et al. ${ }^{4}$ study, confirming the findings of our cumulative meta-analysis that new studies (published after 2017) had little impact on the prevalence estimates. At the same time, all the included studies showed weaknesses and
A study

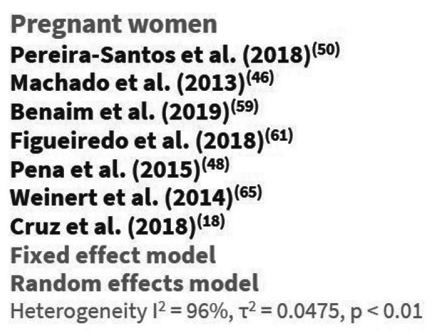

Women of childbearing age

Ferreira et al. (2015) (42)

Menegati et al. (2016) (17)

Pena et al. (2015)(48)

Lopes et al. (2016)(45)

Santos et al. (2017) ${ }^{(53)}$

Chrisostomo et al. (2018) ${ }^{(38)}$

Santos et al. (2019) (54)

Shinjo et al. (2011) $)^{(56)}$

Fixed effect model

Random effects model

Heterogeneity $I^{2}=88 \%, \tau^{2}=0.0129, p<0.01$

Women in adolescence

de Oliveira et al. (2020) ${ }^{(35)}$

Santos et al. (2013)

Fixed effect model

Random effects model

Heterogeneity $\mathrm{l}^{2}=39 \%, \mathrm{~T}^{2}=0.0012, p<0.20$

Postpartum women

Martins et al. (2018)

Dutra et al. (2019)(58)

Simões et al. (2016) $)^{(57)}$

Prado et al. (2015) ${ }^{(3)}$

Fixed effect model

Random effects model

Heterogeneity $l^{2}=99 \%, \tau^{2}=0.1229, p<0.01$

Fixed effect model

Random effects model

Heterogeneity $\left.\right|^{2}=96 \%, \tau^{2}=0.0432, p<0.01$

Residual heterogeneity: $I^{2}=96 \%, p<0.01$
Events Tota

27
7
29
32
46
98
28
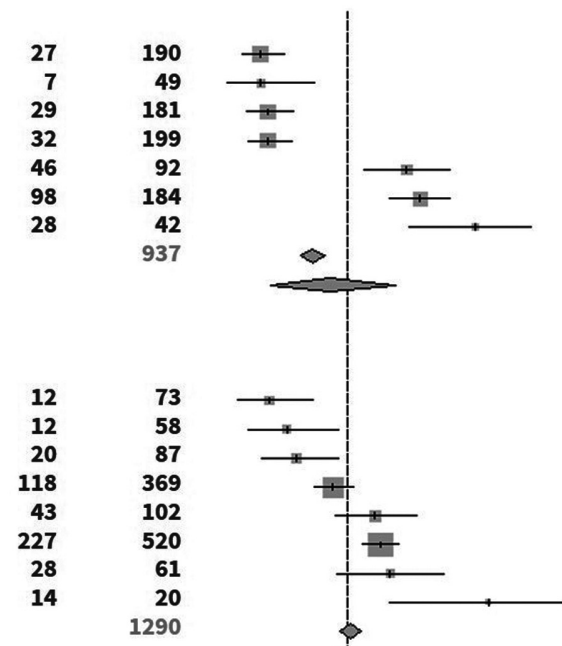

$\begin{array}{ll}26 & 100 \\ 66 & 198 \\ & 298\end{array}$

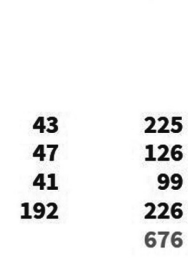

3201

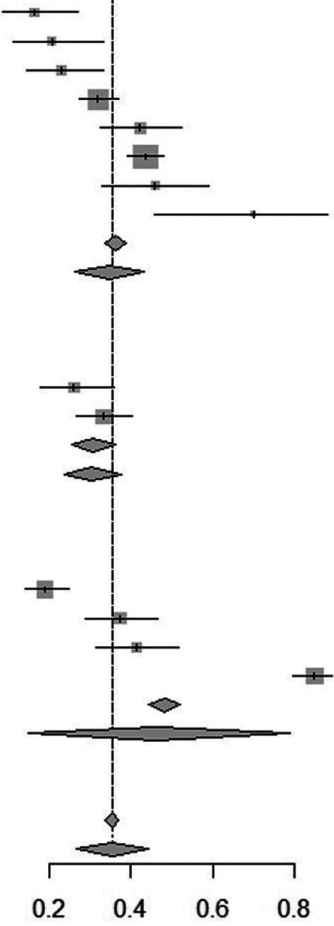

Weight Weight

Proportion 95\%-Cl (fixed) (random)

$\begin{array}{lrr}0.14[0.10 ; 0.20] & 5.9 \% & 4.9 \% \\ 0.14[0.06 ; 0.27] & 1.5 \% & 4.5 \% \\ 0.16[0.11 ; 0.22] & 5.7 \% & 4.9 \% \\ 0.16[0.11 ; 0.22] & 6.2 \% & 4.9 \% \\ 0.50[0.39 ; 0.61] & 2.9 \% & 4.8 \% \\ 0.53[0.46 ; 0.61] & 5.7 \% & 4.9 \% \\ 0.67[0.50 ; 0.80] & 1.3 \% & 4.5 \% \\ 0.27[0.24 ; 0.30] & 29.3 \% & -- \\ 0.31[0.17 ; 0.47] & -- & 33.4 \%\end{array}$

$0.16[0.09 ; 0.27] \quad 2.3 \% \quad 4.7 \%$

$0.21[0.11 ; 0.33] \quad 1.8 \% \quad 4.6 \%$

$0.23[0.15 ; 0.33] \quad 2.7 \% \quad 4.7 \%$

$0.32[0.27 ; 0.37] \quad 11.5 \% \quad 5.0 \%$

$0.42[0.32 ; 0.52] \quad 3.2 \% \quad 4.8 \%$

$0.44[0.39 ; 0.48] \quad 16.2 \% \quad 5.0 \%$

$0.46[0.33 ; 0.59] \quad 1.9 \% \quad 4.6 \%$

$0.70[0.46 ; 0.88] \quad 0.6 \% \quad 3.9 \%$

$0.36[0.34 ; 0.39] \quad 40.3 \% \quad-$

$0.35[0.26 ; 0.43] \quad--\quad 37.4 \%$

$0.26[0.18 ; 0.36] \quad 3.1 \% \quad 4.8 \%$

$0.33[0.27 ; 0.40] \quad 6.2 \% \quad 4.9 \%$

$\begin{array}{lrr}0.31[0.26 ; 0.36] & 9.3 \% & -- \\ 0.30[0.24 ; 0.38] & -- & 9.7 \%\end{array}$

$0.30[0.24 ; 0.38]-2.7 \%$

$0.19[0.14 ; 0.25] \quad 7.0 \% \quad 4.9 \%$

$0.37[0.29 ; 0.46] \quad 3.9 \% \quad 4.8 \%$

$0.41[0.32 ; 0.52] \quad 3.1 \% \quad 4.8 \%$

$0.85[0.80 ; 0.89] \quad 7.1 \% \quad 4.9 \%$

$0.48[0.44 ; 0.52] 21.1 \% \quad--$

$0.46[0.15 ; 0.79] \quad--19.5 \%$

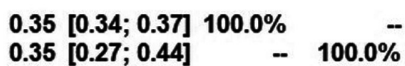

Fig. 1 (A) Vitamin D deficiency in pregnant women, women of childbearing age, women in adolescence, and postpartum women; (B) Vitamin D insufficiency in pregnant women, women of childbearing age, women in adolescence, and postpartum women. 
B

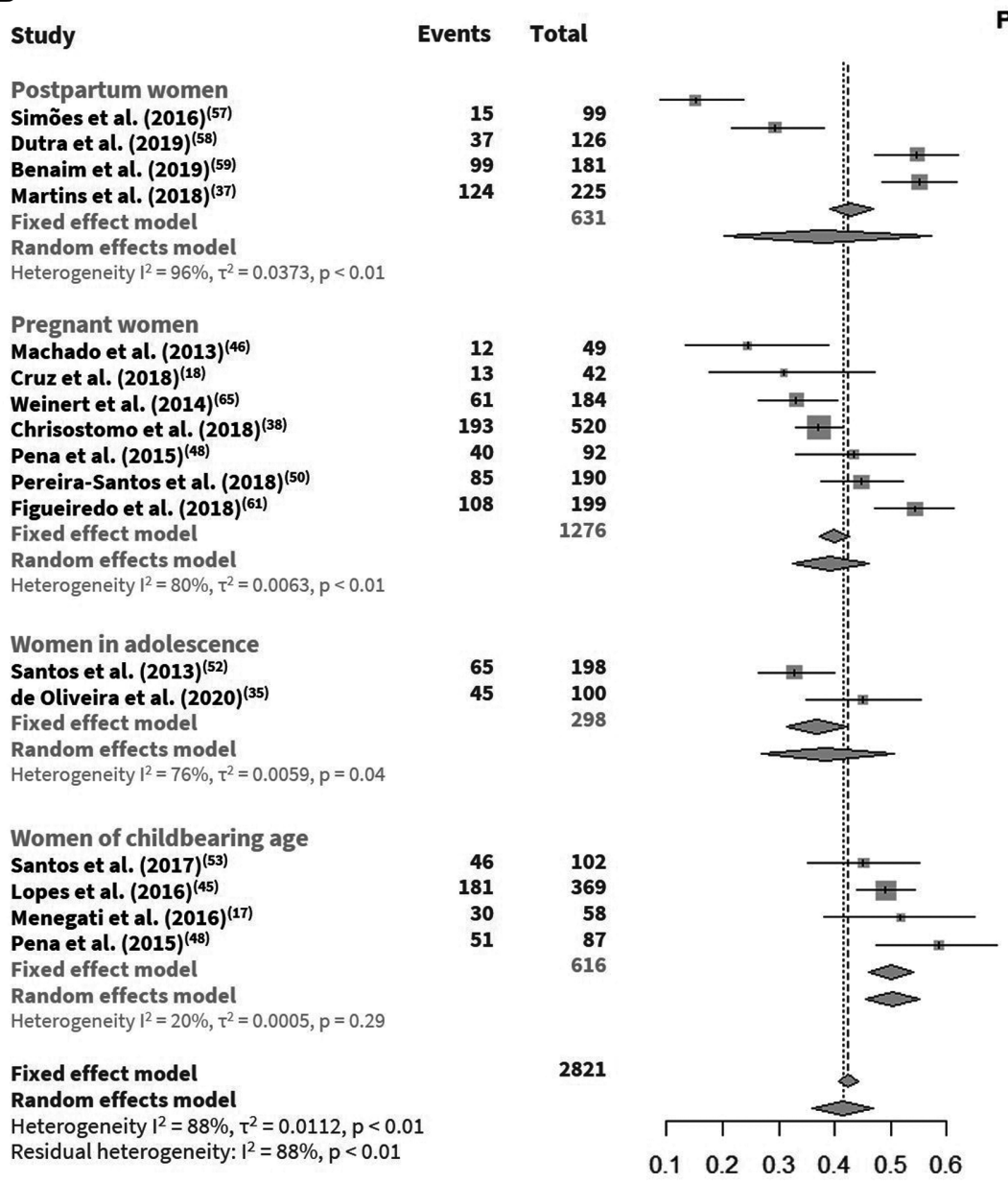

Proportion $\quad 95 \%-\mathrm{Cl}$ Weight $\begin{gathered}\text { Weight } \\ \text { (fixed) (random) }\end{gathered}$

$\begin{array}{rrr}0.15[0.09 ; 0.24] & 3.5 \% & 5.7 \% \\ 0.29[0.22 ; 0.38] & 4.5 \% & 6.0 \% \\ 0.55[0.47 ; 0.62] & 6.4 \% & 6.2 \% \\ 0.55[0.48 ; 0.62] & 8.0 \% & 6.4 \% \\ 0.43[0.39 ; 0.47] & 22.4 \% & -- \\ 0.38[0.20 ; 0.57] & -- & 24.3 \%\end{array}$

$0.24[0.13 ; 0.39] \quad 1.7 \% \quad 4.8 \%$

$0.31[0.18 ; 0.47] \quad 1.5 \% \quad 4.6 \%$

$0.33[0.26 ; 0.40] \quad 6.5 \% \quad 6.3 \%$

$0.37[0.33 ; 0.41] \quad 18.4 \% \quad 6.7 \%$

$0.43[0.33 ; 0.54] \quad 3.3 \% \quad 5.6 \%$

$0.45[0.38 ; 0.52] \quad 6.7 \% \quad 6.3 \%$

$0.54[0.47 ; 0.61] \quad 7.1 \% \quad 6.3 \%$

$0.40[0.37 ; 0.43] \quad 45.2 \%$

$0.39[0.33 ; 0.46] \quad--\quad 40.6 \%$

$0.33[0.26 ; 0.40] \quad 7.0 \% \quad 6.3 \%$

$0.45[0.35 ; 0.55] \quad 3.6 \% \quad 5.7 \%$

$0.37[0.31 ; 0.42] \quad 10.6 \% \quad-$

$0.38[0.27 ; 0.51] \quad--12.0 \%$

$0.45[0.35 ; 0.55] \quad 3.6 \% \quad 5.8 \%$

$0.49[0.44 ; 0.54] \quad 13.1 \% \quad 6.6 \%$

$0.52[0.38 ; 0.65] \quad 2.1 \% \quad 5.1 \%$

$0.59[0.48 ; 0.69] \quad 3.1 \% \quad 5.6 \%$

$0.50[0.46 ; 0.54] \quad 21.8 \% \quad--$

$0.50[0.45 ; 0.55] \quad--\quad 23.0 \%$

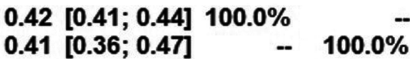

Fig. 1 (Continued)

high heterogeneity, which reduced the confidence on the prevalence rates reported.

Although little variation on the estimates has been added in the last years for women of childbearing age, when considering population subgroups (e.g., adolescents, pregnant women, postpartum women) the uncertainty still exists. For instance, when considered vitamin D deficiency in postpartum women (48\%, 95\% CI 44-52\%, $\mathrm{I}^{2} 99 \%$ ), Martins et al. $^{37}$ identified prevalence of $19 \%$, whereas Prado et al. ${ }^{3}$ described it as $85 \%$. While Martins et al. ${ }^{37}$ included $79 \%$ of dark-skin women (variable associated with deficiency), 64\% using supplement (variable associated with sufficiency), and during spring and summer (variable associated with sufficiency); Prado et al. ${ }^{3}$ included $52 \%$ of white women (variable associated with sufficiency), $97 \%$ using supplement (variable associated with sufficiency), and throughout the year.

Moreover, it was not possible to conduct a robust subgroup analysis to explore the heterogeneity, as well as to identify possible associated factors to deficiency or insufficiency of vitamin $\mathrm{D}$, since most studies did not report the characteristics of the participants, nor population subgroup analysis using common categories. Primary studies should appropriately report the findings according to common subgroups, and minimally, season, skin pigmentation, WHO standardized age group, ${ }^{69}$ and supplement use.

In comparison with international data for inadequate vitamin D levels, our prevalence estimates are lower than estimates for women in Iran (44\% deficiency), ${ }^{70}$ and for women of childbearing age in in Saudi Arabia (77\% deficiency or insufficiency), ${ }^{71}$ but higher than estimates for adolescent girls in India (26\% deficiency). ${ }^{72}$ Several factors can explain the differences between the estimates, such as age, latitude, skin pigmentation, dietary habits, fortification of foods with vitamin D, use of vitamin D supplements, sunlight exposure, and cultural factors. ${ }^{1,73-75}$ To exemplify, Gomes et al. ${ }^{76}$ identified a seriously inadequate intake of vitamin D among Brazilian pregnant women in the primary healthcare network.

It is important to highlight that our systematic review identified several studies evaluating nonpregnant and nonlactating women, which were grouped as women of childbearing age. Notwithstanding, it was noted that many of these women had conditions associated with inadequate levels of vitamin D, such as overweight or obesity, ${ }^{77-79}$ gestational diabetes mellitus, ${ }^{80}$ preeclampsia, ${ }^{81,82}$ cardiovascular disease, ${ }^{83}$ breast cancer, ${ }^{84}$ polycystic ovarian 
A

Study

Events Total

Proportion $\quad 95 \%-\mathrm{Cl}$

$1^{\text {st }}$ trimester

Figueiredo et al. (2018)

Souza et al. $(2019)^{(40)}$

Medeiros et al. (2016) $)^{(64)}$

Cruz et al. (2018)

Fixed effect model

Random effects model

Heterogeneity $\mathrm{I}^{2}=66 \%, \mathrm{~T}^{2}=0.0075, \mathrm{p}=0.03$

$2^{\text {nd }}$ trimester

Figueiredo et al. (2018) $)^{(61)}$

Medeiros et al. (2016) $)^{(64)}$

Cruz et al. (2018)(18)

Souza et al. $(2019)^{(40)}$

Fixed effect model

Random effects model

Heterogeneity $\mathrm{I}^{2}=65 \%, \mathrm{\tau}^{2}=0.0062, p=0.04$

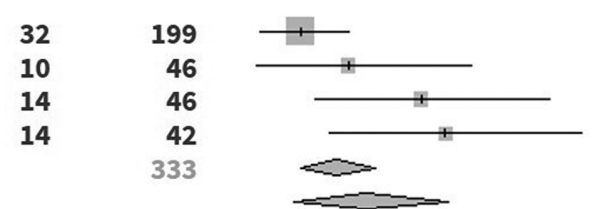

$0.16 \quad[0.11 ; 0.22]$

$0.22[0.11 ; 0.36]$

$0.30 \quad[0.18 ; 0.46]$

$0.33[0.20 ; 0.50]$

$0.20[0.16 ; 0.25]$

$0.24[0.15 ; 0.34]$

$3^{\text {rd }}$ trimester

Figueiredo et al. (2018) ${ }^{(61)}$

Souza et al. (2019) ${ }^{(40)}$

Cruz et al. (2018)(18)

Medeiros et al. (2016) $)^{(64)}$

Fixed effect model

Random effects model

Heterogeneity $\mathrm{I}^{2}=90 \%, \tau^{2}=0.0318, p<0.01$

$\begin{array}{rr}22 & 199 \\ 9 & 46 \\ 9 & 42 \\ 17 & 71\end{array}$

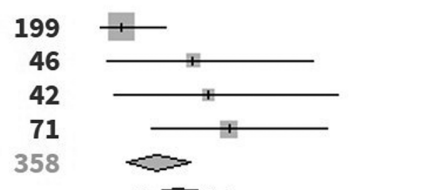

$0.11[0.07 ; 0.16]$

$0.20[0.09 ; 0.34]$

$0.21[0.10 ; 0.37]$

$0.24[0.15 ; 0.36]$

$0.15[0.12 ; 0.19]$

$0.18[0.11 ; 0.26]$

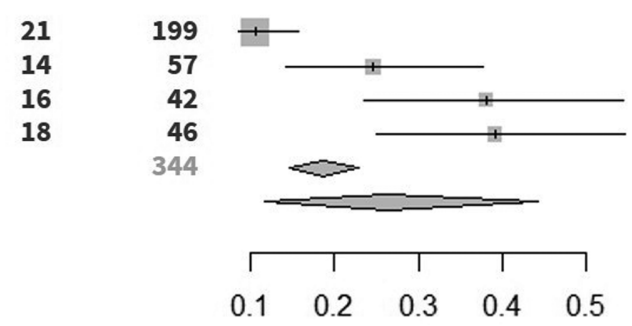

$0.11[0.07 ; 0.16]$

$0.25[0.14 ; 0.38]$

$0.38 \quad[0.24 ; 0.54]$

$0.39[0.25 ; 0.55]$

$0.19[0.15 ; 0.23]$

$0.26[0.12 ; 0.44]$

B

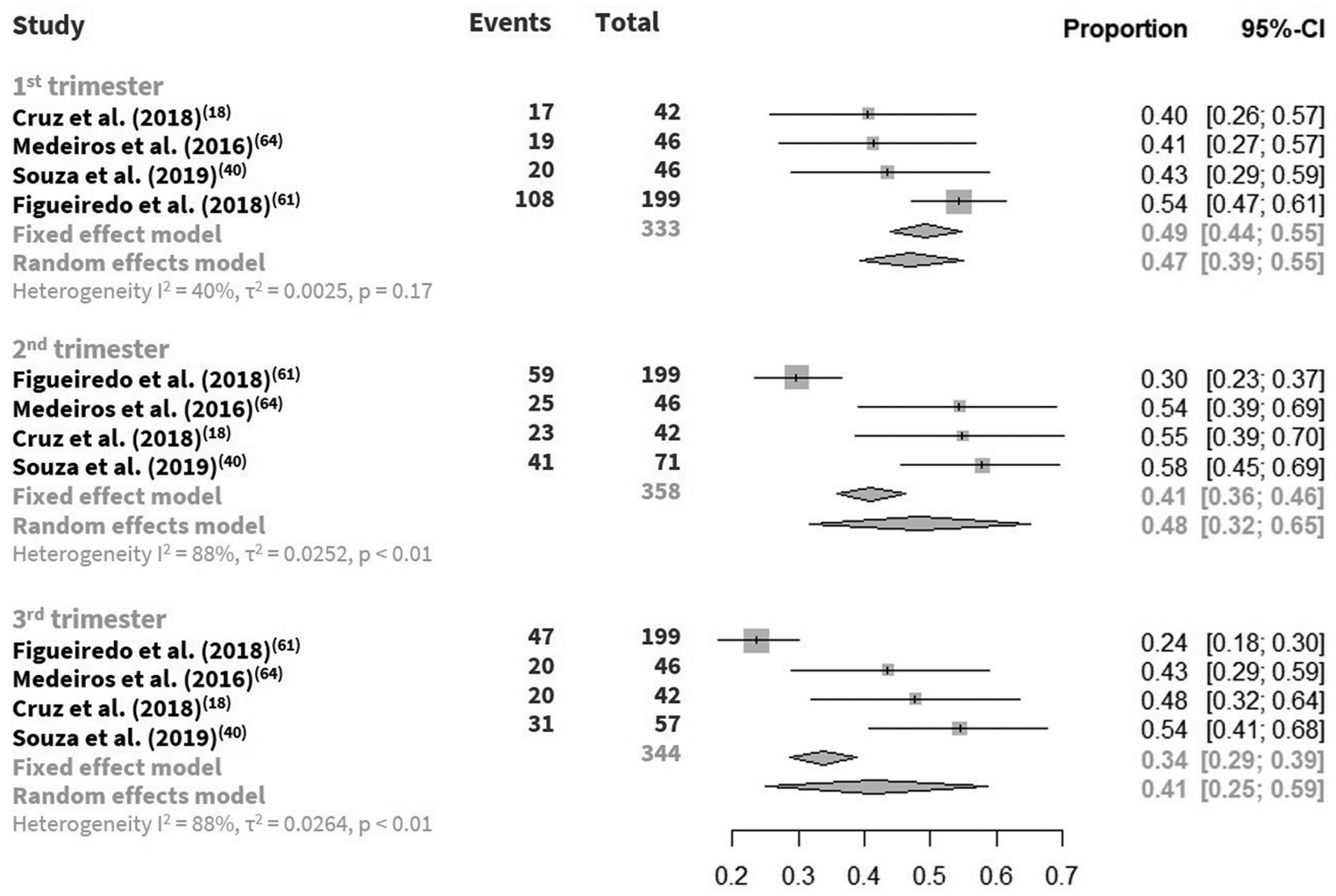

Fig. 2 (A) Vitamin D deficiency along gestational trimesters; (B) Vitamin D insufficiency along gestational trimesters. 
syndrome, ${ }^{85}$ and infertility, ${ }^{86}$ among others, which may overestimate the identified prevalence.

Another important consideration is that despite the variation in cutoff values used by studies to define vitamin $\mathrm{D}$ deficiency, most studies included in this meta-analysis considered the threshold recommended by the US Institute of Medicine $(<50 \mathrm{nmol} / \mathrm{L}$ of $25(\mathrm{OH}) \mathrm{D})$ as opposed to the threshold recommended by the Endocrine Society Practice Guidelines ( $<75 \mathrm{nmol} / \mathrm{L}$ of $25(\mathrm{OH}) \mathrm{D})$. The generally accepted cutoff levels consider the values necessary to ensure optimal effects in the calcium economy and skeletal health, ${ }^{87}$ and studies designed to assess the correlation of clinical responses with clinically relevant vitamin D deficiency suggest that depending on the physiological parameters considered (e.g., pregnancy outcomes, cardiometabolic risk) the results may differ and be even greater than those mentioned above, ${ }^{88-90}$ resulting in the identification of larger populations with vitamin D deficiency. Although it is not possible to be sure about the magnitude of deficiency/insufficiency of vitamin D in some subgroups among Brazilian women, current evidence suggests that this is a public health problem, given the Institute of Medicine's (US) recommended cutoff values. ${ }^{91}$ In this sense, some preventive strategies for adequate vitamin D levels include fish consumption, food fortification, ${ }^{92}$ and advice on moderate sunlight exposure. ${ }^{1,93}$

Among the few countries with specific policies, the United Kingdom and Finland stand out with the recommendation of $10 \mu \mathrm{g}$ of vitamin D daily intake for general population, and the mandatory food fortification programs, respectively. ${ }^{94}$ In pregnant women, conflicting evidence suggests the benefit of supplementation, despite the documented negative clinical, humanistic, and economic impact of the deficiency or insufficiency of vitamin D, mainly, during the first trimester of pregnancy. ${ }^{95}$ The hesitation about the recommendation of supplement intake may be justified by the limited evidence on the safety of vitamin D supplements, which could explain the reason why WHO does not recommend the supplementation during pregnancy as part of routine antenatal care. ${ }^{96}$ Conversely to $\mathrm{WHO}$, the Brazilian consensus recommends supplementation in pregnant women at risk of deficiency. ${ }^{11}$ However, the Brazilian consensus does not recommend generalized vitamin D supplementation for the entire population, while it recommends the assessment of serum levels in obese patients. $^{11}$

Despite several options of vitamin supplements containing vitamin D being available in Brazil, with some of them included in Brazilian National List of Essential Medicines (Rename), ${ }^{97}$ no national policy to prevent vitamin D insufficiency or deficiency in any women subgroup exists. In addition to funding studies to estimate the prevalence of micronutrient deficiencies in women of childbearing age, ${ }^{98} \mathrm{a}$ government policy is needed to avoid vitamin D inadequate levels, as well as excessive intake by self-medication or inappropriate prescription. ${ }^{99}$

As any systematic review, one limitation of this study is that missing studies could exist. To overcome this limitation, extensive gray literature and manual searches to find unpublished and published studies were conducted, having found a few studies not retrieved by electronic searches. Although a high number of studies were identified through manual search, which could be seen as a limitation of the search strategy, one hypothesis is that many studies may not have properly written titles and abstracts, or are not correctly indexed, hindering the automatic search algorithm's ability to retrieve them. Finally, another limitation was the absence of a robust analysis about potential associated factors of inadequate levels of vitamin $\mathrm{D}$, due to the poor reporting of the compiled studies.

\section{Conclusion}

Although the magnitude of the prevalence of inadequate levels of vitamin D is uncertain, the evidence found in the literature suggests a moderate to severe problem with a prevalence of vitamin D deficiency (35\%), insufficiency (42\%), and deficiency or insufficiency (72\%) in women of reproductive age. Future studies about vitamin D levels should consider random probabilistic sampling, appropriate sample sizes and reporting of findings. Furthermore, vitamin D studies should consider estimates according to the season, skin pigmentation, age range standardized by WHO, and use of supplements, to better inform potential health policies.

\section{Contributions}

Lucchetta RC: Conceptualization, Methodology, Writing, Investigation, Visualization, Supervision, Validation; Lemos IH: Writing- Reviewing and Editing, and Investigation; Gini ALR: Writing- Reviewing and Editing, and Investigation; Cavicchioli SA: Writing- Reviewing and Editing, and Investigation; Forgerini M: Visualization, Writing- Reviewing and Editing; Varallo FR: Visualization, Writing- Reviewing and Editing; de Nadai MN: Visualization, Writing- Reviewing and Editing; Fernando Fernandez-Llimós: Visualization, Writing- Reviewing and Editing; Mastroianni P: Conceptualization, Visualization, Validation, Writing- Reviewing and Editing, Project administration.

\section{Funding/Acknowledgments}

This study was funded by the Ministry of Science, Technology, Innovation and Communications, Ministry of Health of Brazil - MoH, and the National Council for Scientific and Technological Development - CNPq (n. 26/2019, process 4426122019-2). Additionally, this study was financed in part by the Coordenação de Aperfeiçoamento de Pessoal de Nível Superior - Brasil (CAPES) Finance Code 001, and the São Paulo Research Foundation (FAPESP) [grant number 2018/07501-9].

\section{Conflict to Interests}

The authors have no conflict of interests to declare.

\section{References}

1 van Schoor N, Lips P. Global overview of Vitamin D status. Endocrinol Metab Clin North Am. 2017;46(04):845-870. Doi: 10.1016/j.ecl.2017.07.002 
2 Saraf R, Morton SM, Camargo CA Jr, Grant CC. Global summary of maternal and newborn vitamin D status - a systematic review. Matern Child Nutr. 2016;12(04):647-668. Doi: 10.1111/ mcn. 12210

3 do Prado MR, Oliveira FdeC, Assis KF, Ribeiro SA, Prado Jr. PP, Sant'Ana LF, et al. Prevalência de deficiência de vitamina D e fatores associados em mulheres e seus recém-nascidos no período pós-parto. Rev Paul Pediatr. 2015;33(03):287-294. Doi: 10.1016/j.rpped.2015.01.006

4 Pereira-Santos M, Santos JYGD, Carvalho GQ, Santos DBD, Oliveira AM. Epidemiology of vitamin D insufficiency and deficiency in a population in a sunny country: Geospatial meta-analysis in Brazil. Crit Rev Food Sci Nutr. 2019;59(13):2102-2109. Doi: 10.1080/10408398.2018.1437711

5 Bischoff-Ferrari HA, Giovannucci E, Willett WC, Dietrich T, Dawson-Hughes B. Estimation of optimal serum concentrations of 25hydroxyvitamin D for multiple health outcomes. Am J Clin Nutr. 2006;84(01):18-28. Doi: 10.1093/ajcn/84.1.18

6 Holick MF. High prevalence of vitamin D inadequacy and implications for health. Mayo Clin Proc. 2006;81(03):353-373. Doi: 10.4065/81.3.353

7 Wortsman J, Matsuoka LY, Chen TC, Lu Z, Holick MF. Decreased bioavailability of vitamin D in obesity. Am J Clin Nutr. 2000;72 (03):690-693. Doi: 10.1093/ajcn/72.3.690

8 Pittas AG, Harris SS, Stark PC, Dawson-Hughes B. The effects of calcium and vitamin D supplementation on blood glucose and markers of inflammation in nondiabetic adults. Diabetes Care. 2007;30(04):980-986. Doi: 10.2337/dc06-1994

9 DeLuca HF. Overview of general physiologic features and functions of vitamin D. Am J Clin Nutr. 2004;80(6, Suppl)1689S-1696S. Doi: 10.1093/ajcn/80.6.1689S

10 Santaolalla A, Beckmann K, Kibaru J, Josephs D, Van Hemelrijck M, Irshad S. Association Between Vitamin D and Novel SARS-CoV-2 respiratory dysfunction - a scoping review of current evidence and its implication for COVID-19 pandemic. Front Physiol. 2020; 11:564387. Doi: 10.3389/fphys.2020.564387

11 Maeda SS, Borba VZ, Camargo MB, Silva DM, Borges JL, Bandeira F, et al.Brazilian Society of Endocrinology and Metabology (SBEM) Recommendations of the Brazilian Society of Endocrinology and Metabology (SBEM) for the diagnosis and treatment of hypovitaminosis D. Arq Bras Endocrinol Metabol. 2014;58(05):411-433

12 Siqueira TW, Araujo Júnior E, Mattar R, Daher S. Assessment of polymorphism of the VDR gene and serum Vitamin D values in gestational diabetes mellitus. Rev Bras Ginecol Obstet. 2019;41 (07):425-431. Doi: 10.1055/s-0039-1693678

13 van der Pligt P, Willcox J, Szymlek-Gay EA, Murray E, Worsley A, Daly RM. Associations of maternal Vitamin D deficiency with pregnancy and neonatal complications in developing countries: a systematic review. Nutrients. 2018;10(05):640. Doi: 10.3390/ nu10050640

14 Wei SQ Qi HP, Luo ZC, Fraser WD. Maternal vitamin D status and adverse pregnancy outcomes: a systematic review and metaanalysis. J Matern Fetal Neonatal Med. 2013;26(09):889-899. Doi: $10.3109 / 14767058.2013 .765849$

15 Lapillonne A. Vitamin D deficiency during pregnancy may impair maternal and fetal outcomes. Med Hypotheses. 2010;74(01): 71-75. Doi: 10.1016/j.mehy.2009.07.054

16 Robinson M, Whitehouse AJ, Newnham JP, Gorman S, Jacoby P, Holt BJ, et al. Low maternal serum vitamin D during pregnancy and the risk for postpartum depression symptoms. Arch Women Ment Health. 2014;17(03):213-219. Doi: 10.1007/s00737-0140422-y

17 Menegati GC, de Oliveira LC, Santos AL, Cohen L, Mattos F, Mendonça LM, et al. Nutritional status, body composition, and bone health in women after bariatric surgery at a University Hospital in Rio de Janeiro. Obes Surg. 2016;26(07):1517-1524. Doi: $10.1007 / \mathrm{s} 11695-015-1910-5$
18 Cruz S, de Matos AC, da Cruz SP, Pereira S, Saboya C, Ramalho A. Maternal anthropometry and its relationship with the nutritional status of Vitamin D, calcium, and parathyroid hormone in pregnant women after Roux-en-Y gastric bypass. Obes Surg. 2018;28 (10):3116-3124. Doi: 10.1007/s11695-018-3331-8

19 Cruz S, de Matos AC, da Cruz SP, Pereira S, Saboya C, Ramalho A. Non-pregnant women have a lower Vitamin D than pregnant women after gastric bypass. Obes Surg. 2020;30(07):2558-2565. Doi: $10.1007 / \mathrm{s} 11695-020-04512-5$

20 Stroup DF, Berlin JA, Morton SC, Olkin I, Williamson GD, Rennie D, et al. Meta-analysis of observational studies in epidemiology: a proposal for reporting. Meta-analysis Of Observational Studies in Epidemiology (MOOSE) group. JAMA. 2000;283(15):2008-2012. Doi: $10.1001 /$ jama.283.15.2008

21 Lizarondo L, Stern C, Carrier J, Godfrey C, Rieger KL, Salmond S, et al. Chapter 8: mixed methods systematic reviews. In: Aromataris E, Munn Z, editors. JBI manual for evidence synthesis [Internet]. Adelaide: JBI; 2017 [cited 2020 Dec 12]. Available from: https://jbiglobal-wiki.refined.site/space/MANUAL/3283910764/Chapter $+8 \%$ $3 \mathrm{~A}+$ Mixed + methods + systematic + reviews

22 Munn Z, Moola S, Lisy K, Riitano D, Tufanaru C. Chapter 5: systematic reviews of prevalence and incidence. In: Aromataris E, Munn Z, editors. JBI manual for evidence synthesis [Internet]. Adelaide: JBI; 2017 [cited 2020 Dec 12]. Available from: https:// jbi-global-wiki.refined.site/space/MANUAL/3283910689/

Chapter $+5 \% 3 \mathrm{~A}+$ Systematic + reviews + of + prevalence + and +incidence

23 Lucchetta R. Protocol: Prevalence of micronutrient deficiencies in women of childbearing age in Brazil: systematic review and metaanalysis [Internet]. OSF. 2020 [cited 2021 Jun 22]. Available from: https://doi.org/10.17605/OSF.IO/J9QMH

24 Hane PJ. Newsbreaks: Elsevier announces Scopus service [Internet]. Information Today. 2004 Mar 15 [cited 2017 Sep 13]. Available from: http://www.infotoday.com/newsbreaks/nb040315-1.shtml

25 Munn Z, Stern C, Aromataris E, Lockwood C, Jordan Z. What kind of systematic review should I conduct? A proposed typology and guidance for systematic reviewers in the medical and health sciences. BMC Med Res Methodol. 2018;18(01):5. Doi: 10.1186/ s12874-017-0468-4

$26 \mathrm{R}$ Core Team. The $\mathrm{R}$ project for statistical computing [Internet]. Vienna: R Foundation; 2020 [cited 2020 Dec 12]. Available from: https://www.r-project.org/

27 Wickham H, Hester J, François R. Readr: Read rectangular text data [Internet]. 2017 [cited 2020 Dec 12]. Available from: https:// cran.r-project.org/package $=$ readr

28 Schwarzer G. Meta: an R package for meta-analysis. R News [Internet]. 2007 [cited 2020 Dec 12];7(3):40-5. Available from: https://www.researchgate.net/profile/Guido-Schwarzer/publication/285729385_meta_An_R_Package_for_Meta-Analysis/ meta: An R Package for Meta-Analysis

29 Mahadev S, Laszkowska M, Sundström J, Björkholm M, Lebwohl B, Green $\mathrm{PH}$, et al. Prevalence of celiac disease in patients with iron deficiency anemia: a systematic review with meta-analysis. Gastroenterology. 2018;155(02):374-382.e1. Doi: 10.1053/j.gastro.2018.04.016

30 Munn Z, Moola S, Lisy K, Riitano D, Tufanaru C. Methodological guidance for systematic reviews of observational epidemiological studies reporting prevalence and cumulative incidence data. Int J Evid-Based Healthc. 2015;13(03):147-153. Doi: 10.1097/ XEB.0000000000000054

31 Migliavaca CB, Stein C, Colpani V, Munn Z, Falavigna MPrevalence Estimates Reviews - Systematic Review Methodology Group (PERSyst) Quality assessment of prevalence studies: a systematic review. J Clin Epidemiol. 2020;127:59-68. Doi: 10.1016/j.jclinepi.2020.06.039

32 Santos Araújo EPD, Queiroz DJM, Neves JPR, Lacerda LM, Gonçalves MDCR, Carvalho AT. Prevalence of hypovitaminosis D and associated factors in adolescent students of a capital of 
northeastern Brazil. Nutr Hosp. 2017;34(05):1416-1423. Doi: 10.20960/nh.1097

33 Queiroz DJ. Relação entre insuficiência/deficiência da vitamina D, inflamação e estresse oxidativo em adolescentes escolares com excesso de peso [dissertação]. João Pessoa: Universidade Federal da Paraíba; 2016

34 Queiroz DJM, Silva AS, Diniz ADS, Carvalho AT, Araújo EP, Neves JP, et al. Vitamin D insufficiency/deficiency and its association with cardiometabolic risk factors in Brazilian adolescents. Nutr Hosp. 2019;36(01):142-148. Doi: 10.20960/nh.1884

35 de Oliveira CL, Cureau FV, Cople-Rodrigues CDS, Giannini DT, Bloch KV, Kuschnir MC, et al. Prevalence and factors associated with hypovitaminosis D in adolescents from a sunny country: Findings from the ERICA survey. J Steroid Biochem Mol Biol. 2020; 199:105609. Doi: 10.1016/j.jsbmb.2020.105609

36 dos Santos LA, de Azeredo VB, Eloy Chaves Barbosa D, Augusta de Sá $S$ Seric ion level and its relationship with the symptoms of premenstrual syndrome in young women. Nutr Hosp. 2013;28 (06):2194-2200

37 Martins MEP, Esmeraldo CUP, Sabiá JPD, Carvalho JHL, SuanoSouza FI, Sarni ROS. Vitamin D postpartum concentrations: relationship with nutritional condition and morbidities during pregnancy. J Pregnancy. 2018;2018:1070528. Doi: 10.1155/2018/ 1070528

38 Chrisostomo KR, Skare TL, Kulak J Jr, Urbanetz AA, Chrisostomo ER, Nisihara R. The prevalence and clinical associations of hypovitaminosis D in pregnant women from Brazil. Int J Gynaecol Obstet. 2018;143(01):66-70. Doi: 10.1002/ijgo.12575

39 Duran de Campos C, Dalcanale L, Pajecki D, Garrido AB Jr, Halpern A. Calcium intake and metabolic bone disease after eight years of Roux-en-Y gastric bypass. Obes Surg. 2008;18(04):386-390. Doi: 10.1007/s11695-007-9393-7

40 Souza JR, Silva TS, Figueredo ED. Hypovitaminosis D in pregnancy: is it a public health issue? Rev Bras Saúde Matern Infant. 2019;19 (01):197-205. Doi: 10.1590/1806-93042019000100011

41 Delmonico L, Costa MASM, Azevedo CM, Silvestre RT, Scherrer LR, Ornellas MH, et al. Low levels of Vitamin D in a cohort of women with impalpable breast lesions from Rio de Janeiro/Brazil. Asian Pac J Cancer Prev. 2018;19(11):3087-3092. Doi: 10.31557/ APJCP.2018.19.11.3087

42 Ferreira TdaS, Rocha TM, Klein MR, Sanjuliani AF. Vitamin d deficiency is associated with insulin resistance independent of intracellular calcium, dietary calcium and serum levels of parathormone, calcitriol and calcium in premenopausal women. Nutr Hosp. 2015;31(04):1491-1498. Doi: 10.3305/ nh.2015.31.4.8490

43 Flauzino T, Simao ANC, de Almeida ERD, Morimoto HK, Oliveira $\mathrm{SR}$, Alfieri DF, et al. Association between Vitamin D status, oxidative stress biomarkers and viral load in human immunodeficiency virus type 1 infection. Curr HIV Res. 2017;15(05): 336-344. Doi: 10.2174/1570162X15666171005170227

44 Lopes MP, Giudici KV, Marchioni DM, Fisberg RM, Martini LA. Relationships between $n-3$ polyunsaturated fatty acid intake, serum 25 hydroxyvitamin $\mathrm{D}$, food consumption, and nutritional status among adolescents. Nutr Res. 2015;35(08):681-688. Doi: 10.1016/j.nutres.2015.05.018

45 Lopes VM, Lopes JR, Brasileiro JP, Oliveira I, Lacerda RP, Andrade MR, et al. Highly prevalence of vitamin D deficiency among Brazilian women of reproductive age. Arch Endocrinol Metab. 2017;61(01):21-27. Doi: 10.1590/2359-3997000000216

46 Machado RH, Bonafe S, Castelo A, Patin RV. Vitamin profile of pregnant women living with HIV/AIDS. ESPEN J. 2013;8(03): e108-e112. Doi: 10.1016/j.clnme.2013.03.002

47 Mendes MM, Hart KH, Lanham-New SA, Botelho PB. Association between 25 -Hydroxyvitamin D, parathyroid hormone, Vitamin D and calcium intake, and bone density in healthy adult women: a cross-sectional analysis from the D-SOL study. Nutrients. 2019;11 (06):1267. Doi: 10.3390/nu11061267
48 Pena HR, de Lima MC, Brandt KG, de Antunes MM, da Silva GA. Influence of preeclampsia and gestational obesity in maternal and newborn levels of vitamin D. BMC Pregnancy Childbirth. 2015;15 (01):112. Doi: 10.1186/s12884-015-0547-7

49 Pereira-Santos M. Determinantes socioambientais das concentrações séricas de vitamina D em gestantes [dissertação]. Salvador: Universidade Federal da Bahia; 2014

50 Pereira-Santos M, Queiroz Carvalho G, David Couto R, Barbosa Dos Santos D, Marlucia Oliveira A. Vitamin D deficiency and associated factors among pregnant women of a sunny city in Northeast of Brazil. Clin Nutr ESPEN. 2018;23:240-244. Doi: 10.1016/j. clnesp.2017.09.007

51 Peters BS, dos Santos LC, Fisberg M, Wood RJ, Martini LA. Prevalence of vitamin D insufficiency in Brazilian adolescents. Ann Nutr Metab. 2009;54(01):15-21. Doi: 10.1159/000199454

52 Santos BR, Mascarenhas LP, Boguszewski MC, Spritzer PM. Variations in the vitamin D-binding protein (DBP) gene are related to lower 25-hydroxyvitamin D levels in healthy girls: a cross-sectional study. Horm Res Paediatr. 2013;79(03):162-168. Doi: $10.1159 / 000348847$

53 Santos BR, Lecke SB, Spritzer PM. Genetic variant in vitamin Dbinding protein is associated with metabolic syndrome and lower 25-hydroxyvitamin D levels in polycystic ovary syndrome: A cross-sectional study. PLoS One. 2017;12(03):e0173695. Doi: 10.1371/journal.pone.0173695

54 Santos BR, Costa NC, Silva TR, Oppermann K, Magalhães JA, Casanova G, et al. Prevalence of vitamin D deficiency in women from southern Brazil and association with vitamin D-binding protein levels and GC-DBP gene polymorphisms. PLoS One. 2019; 14(12):e0226215. Doi: 10.1371/journal.pone.0226215

55 Schtscherbyna A, Gouveia C, Pinheiro MF, Luiz RR, Farias ML, Machado ES. Vitamin D status in a Brazilian cohort of adolescents and young adults with perinatally acquired human immunodeficiency virus infection. Mem Inst Oswaldo Cruz. 2016;111(02): 128-133. Doi: 10.1590/0074-02760150403

56 Shinjo SK, Bonfá E, de Falco Caparbo V, Pereira RM. Low bone mass in juvenile onset sclerosis systemic: the possible role for 25hydroxyvitamin D insufficiency. Rheumatol Int. 2011;31(08): 1075-1080. Doi: 10.1007 /s00296-010-1421-6

57 Simões FF, Castro NP, Euclydes VV, Luzia LA, Paiva AA, Rondó PH. Maternal adiposity and maternal and cord blood concentrations of vitamin D. [25(OH)D3]Clin Nutr Exp. 2016;9:3-12. Doi: 10.1016/j.yclnex.2016.08.001

58 Dutra LV, Affonso-Kaufman FA, Cafeo FR, Kassai MS, Barbosa CP, Santos Figueiredo FW, et al. Association between vitamin D plasma concentrations and VDR gene variants and the risk of premature birth. BMC Pregnancy Childbirth. 2019;20(01):3. Doi: 10.1186/s12884-019-2671-2

59 Benaim C, Cocate PG, de Barros EG, Alves-Santos NH, Figueiredo $A C$, Franco-Sena AB, et al. Longitudinal association of 25-hydroxyvitamin $\mathrm{D}$ with adipokines and markers of glucose metabolism among Brazilian pregnant women. Br J Nutr. 2019;121(01): 42-54. Doi: 10.1017/S0007114518003057

60 Lepsch J, Eshriqui I, Farias DR, Vaz JS, Figueiredo AC, Adegboye AR, et al. Association between early pregnancy vitamin $D$ status and changes in serum lipid profiles throughout pregnancy. Metabolism. 2017;70:85-97. Doi: 10.1016/j.metabol.2017.02.004

61 Figueiredo ACC, Cocate PG, Adegboye ARA, Franco-Sena AB, Farias $\mathrm{DR}$, deCastro MBT, et al. Changes in plasma concentrations of 25hydroxyvitamin $\mathrm{D}$ and 1,25-dihydroxyvitamin $\mathrm{D}$ during pregnancy: a Brazilian cohort. Eur J Nutr. 2018;57(03):1059-1072. Doi: 10.1007/s00394-017-1389-z

62 Cunha Figueiredo AC, Trujillo J, Freitas-Vilela AA, Franco-Sena AB, Rebelo F, Cunha GM, et al. Association between plasma concentrations of vitamin D metabolites and depressive symptoms throughout pregnancy in a prospective cohort of Brazilian women. J Psychiatr Res. 2017;95:1-8. Doi: 10.1016/j.jpsychires.2017.07.009 
63 Figueiredo ACC, Carrilho TRB, Batalha MA, Farias DR, Barros EG, Kac G. Association between vitamin D status during pregnancy and total gestational weight gain and postpartum weight retention: a prospective cohort. Eur J Clin Nutr. 2020;74(01):126-134. Doi: 10.1038/s41430-019-0465-2

64 Medeiros M, Matos AC, Pereira SE, Saboya C, Ramalho A. Vitamin D and its relation with ionic calcium, parathyroid hormone, maternal and neonatal characteristics in pregnancy after roux-en-Y gastric bypass. Arch Gynecol Obstet. 2016;293(03):539-547. Doi: 10.1007/s00404-015-3861-4

65 Weinert LS, Reichelt AJ, Schmitt LR, Boff R, Oppermann ML, Camargo JL, et al. Serum vitamin D insufficiency is related to blood pressure in diabetic pregnancy. Am J Hypertens. 2014;27 (10):1316-1320. Doi: 10.1093/ajh/hpu043

66 Weinert LS, Reichelt AJ, Schmitt LR, Boff R, Oppermann ML, Camargo JL, et al. Vitamin D deficiency increases the risk of adverse neonatal outcomes in gestational diabetes. PLoS One. 2016;11(10):e0164999. Doi: 10.1371/journal.pone.0164999

67 da Cruz SP, Matos A, Pereira S, Saboya C, da Cruz SP, Ramalho A. Roux-en-Y gastric bypass aggravates Vitamin A deficiency in the mother-child group. Obes Surg. 2018;28(01):114-121. Doi: 10.1007/s11695-017-2791-6

68 da Rosa CL, Dames Olivieri Saubermann AP, Jacqueline J, Pereira SE, Saboya C, Ramalho A. Routine supplementation does not warrant the nutritional status of vitamin d adequate after gastric bypass Roux-en-Y. Nutr Hosp. 2013;28(01):169-172. Doi: 10.3305/nh.2013.28.1.6166

69 Ahmad OB, Boschi-Pinto C, Lopez AD, Murray CJ, Lozano R, Inoue M. Age standardization of rates: a new WHO standard [Internet]. Geneva: WHO; 2001

70 Vatandost S, Jahani M, Afshari A, Amiri MR, Heidarimoghadam R, Mohammadi Y. Prevalence of vitamin D deficiency in Iran: A systematic review and meta-analysis. Nutr Health. 2018;24(04): 269-278. Doi: $10.1177 / 0260106018802968$

71 Alzaheb RA. The prevalence of hypovitaminosis D and its associated risk factors among women of reproductive age in Saudi Arabia: a systematic review and meta-analysis. Clin Med Insights Womens Health. 2018;11:X18767884

72 Jeyakumar A, Shinde V. A systematic review and meta-analysis of prevalence of vitamin $\mathrm{D}$ deficiency among adolescent girls in selected Indian states. Nutr Health. 2019;25(01):61-70. Doi: 10.1177/0260106018805360

73 Mithal A, Wahl DA, Bonjour JP, Burckhardt P, Dawson-Hughes B, Eisman JA, et al.IOF Committee of Scientific Advisors (CSA) Nutrition Working Group. Global vitamin D status and determinants of hypovitaminosis D. Osteoporos Int. 2009;20(11):1807-1820. Doi: 10.1007/s00198-009-0954-6

74 Aji AS, Yerizel E, Desmawati, Lipoeto NI. The association between lifestyle and maternal vitamin D during pregnancy in West Sumatra, Indonesia. Asia Pac J Clin Nutr. 2018;27(06):1286-1293. Doi: 10.6133/apjcn.201811_27(6).0016

75 Bonatto S, Vieira Paniz VM, de Freitas Dutra C, Dutra BV, Henn RL. Serum 25-hydroxyvitamin D concentration among users of a referral outpatient unit for vascular diseases and associated factors. Clin Nutr ESPEN. 2021;42:299-306. Doi: 10.1016/j. clnesp.2021.01.017

76 Gomes CB, Malta MB, Corrente JE, Benício MH, Carvalhaes MA. Alta prevalência de inadequação da ingestão dietética de cálcio e vitamina D em duas coortes de gestantes. Cad Saude Publica. 2016;32(12):e00127815. Doi: 10.1590/0102-311X00127815

77 Hajhashemy Z, Shahdadian F, Ziaei R, Saneei P. Serum vitamin D levels in relation to abdominal obesity: A systematic review and dose-response meta-analysis of epidemiologic studies. Obes Rev. 2021;22(02):e13134. Doi: 10.1111/obr.13134

78 de Oliveira LF, de Azevedo LG, da Mota Santana J, de Sales LPC, Pereira-Santos M. Obesity and overweight decreases the effect of vitamin D supplementation in adults: systematic review and meta-analysis of randomized controlled trials. Rev Endocr
Metab Disord. 2020;21(01):67-76. Doi: 10.1007/s11154-01909527-7

79 Jiang S, Zhu Q, Mai M, Yang W, Du G. Vitamin B and vitamin D as modulators of gut microbiota in overweight individuals. Int J Food Sci Nutr. 2020;71(08):1001-1009. Doi: 10.1080/09637486. 2020.1748580

80 Wang L, Zhang C, Song Y, Zhang Z. Serum vitamin D deficiency and risk of gestational diabetes mellitus: a meta-analysis. Arch Med Sci. 2020;16(04):742-751. Doi: 10.5114/aoms.2020.94433

81 Aguilar-Cordero MJ, Lasserrot-Cuadrado A, Mur-Villar N, LeónRíos XA, Rivero-Blanco T, Pérez-Castillo IM. Vitamin D, preeclampsia and prematurity: A systematic review and meta-analysis of observational and interventional studies. Midwifery. 2020; 87:102707. Doi: 10.1016/j.midw.2020.102707

82 Fogacci S, Fogacci F, Banach M, Michos ED, Hernandez AV, Lip GY, et al.Lipid and Blood Pressure Meta-analysis Collaboration (LBPMC) Group. Vitamin D supplementation and incident preeclampsia: A systematic review and meta-analysis of randomized clinical trials. Clin Nutr. 2020;39(06):1742-1752. Doi: 10.1016/j. clnu.2019.08.015

83 Ferraz GC, Andrade RR, Reis FMP, OliveiraNeto OB, Omena CO, Jucá $\mathrm{MJ}$, et al. Association between vitamin D and cardioprotection in adult patients. Rev Assoc Med Bras (1992). 2020;66(10):1444-1448. Doi: 10.1590/1806-9282.66.10.1444

84 Hossain S, Beydoun MA, Beydoun HA, Chen X, Zonderman AB, Wood RJ. Vitamin D and breast cancer: A systematic review and meta-analysis of observational studies. Clin Nutr ESPEN. 2019; 30:170-184. Doi: 10.1016/j.clnesp.2018.12.085

85 Pergialiotis V, Karampetsou N, Panagopoulos P, Trakakis E, Papantoniou N. The effect of Vitamin D supplementation on hormonal and glycaemic profile of patients with PCOS: A meta-analysis of randomised trials. Int J Clin Pract. 2017;71(06):e12957. Doi: 10.1111/ijcp. 12957

86 Fichera M, Török P, Tesarik J, Della Corte L, Rizzo G, Garzon S, et al. Vitamin D, reproductive disorders and assisted reproduction: evidences and perspectives. Int J Food Sci Nutr. 2020;71(03): 276-285. Doi: 10.1080/09637486.2019.1661978

87 Holick MF, Chen TC. Vitamin D deficiency: a worldwide problem with health consequences. Am J Clin Nutr. 2008;87(04):1080S-1086S. Doi: 10.1093/ajcn/87.4.1080S

88 Katrinaki M, Kampa M, Margioris A, Castanas E, Malliaraki N. Vitamin D levels in a large Mediterranean cohort: reconsidering normal cut-off values. Hormones (Athens). 2016;15(02): 205-223. Doi: 10.14310/horm.2002.1674

89 Rostami M, Simbar M, Amiri M, Bidhendi-Yarandi R, Hosseinpanah F, Ramezani Tehrani F. The optimal cut-off point of vitamin $D$ for pregnancy outcomes using a generalized additive model. Clin Nutr. 2021;40(04):2145-2153. Doi: 10.1016/j.clnu.2020. 09.039

90 Milagres LC, Filgueiras MS, Rocha NP, Suhett LG, de Albuquerque FM, Juvanhol LL, et al. Cutoff point estimation for serum vitamin D concentrations to predict cardiometabolic risk in Brazilian children. Eur J Clin Nutr. 2020;74(12):1698-1706. Doi: 10.1038/ s41430-020-0624-5

91 Ross AC, Manson JE, Abrams SA, Aloia JF, Brannon PM, Clinton SK, et al. The 2011 report on dietary reference intakes for calcium and vitamin D from the Institute of Medicine: what clinicians need to know. J Clin Endocrinol Metab. 2011;96(01):53-58. Doi: 10.1210/ jc.2010-2704

92 Weir RR, Johnston M, Lowis C, Fearon AM, Stewart S, Strain JJ, et al. Vitamin $D_{3}$ content of cows' milk produced in Northern Ireland and its efficacy as a vehicle for vitamin D fortification: a UK model. Int J Food Sci Nutr. 2021;72(04):447-455. Doi: 10.1080/09637486.2020.1837743

93 Aguilar-Shea AL. Vitamin D, the natural way. Clin Nutr ESPEN. 2021;41:10-12. Doi: 10.1016/j.clnesp.2020.12.001

94 Mendes MM, Charlton K, Thakur S, Ribeiro H, Lanham-New SA. Future perspectives in addressing the global issue of vitamin D 
424 Deficiency and Insufficiency of Vitamin D in Women of Childbearing Age Lucchetta et al.

deficiency. Proc Nutr Soc. 2020;79(02):246-251. Doi: 10.1017/ S0029665119001538

95 Palacios C, Kostiuk LK, Peña-Rosas JP. Vitamin D supplementation for women during pregnancy. Cochrane Database Syst Rev. 2019; 7(07):CD008873. Doi: 10.1002/14651858.CD008873.pub4

96 World Health Organization. Vitamin D supplementation in pregnant women [Internet]. Geneva: WHO; 2012 [cited 2021 Jan 2]. Available from: https://apps.who.int/iris/bitstream/handle/ 10665/85313/9789241504935_eng.pdf?ua=1

97 Ministério da Saúde. Secretaria de Ciência, Tecnologia, Inovação e Insumos Estratégicos em Saúde. Departamento de Assistência Farmacêutica e Insumos Estratégicos. Relação nacional de medicamentos essenciais - Rename 2020 [Internet]. Brasília (DF): Ministério da Saúde; 2020 [cited 2021 Jan 25]. Available from: http://bvsms.saude.gov.-

br/bvs/publicacoes/relacao_medicamentos_rename_2020.pdf

98 Ministério da Saúde. Secretaria de Ciência, Tecnologia e Insumos Estratégicos. Conselho Nacional de Desenvolvimento Científico e Tecnológico. Chamada MS-SCTIE-Decit / CNPq No 26/2019-Pesquisas em Alimentação e Nutrição [Internet]. 2019 [cited 2021 Jan 20]. Available from: http://www.ppgcardiologia.com.br/wp-content/uploads/2019/09/Chamada_26_2019_Pesquisas_ Alimentacao_Nutricao-1.pdf

99 Moyersoen I, Lachat C, Cuypers K, Ridder K, Devleesschauwer B, Tafforeau J, et al. Do current fortification and supplementation programs assure adequate intake of fat-soluble vitamins in Belgian infants, toddlers, pregnant women, and lactating women? Nutrients. 2018;10(02):223. Doi: 10.3390/nu10020223 